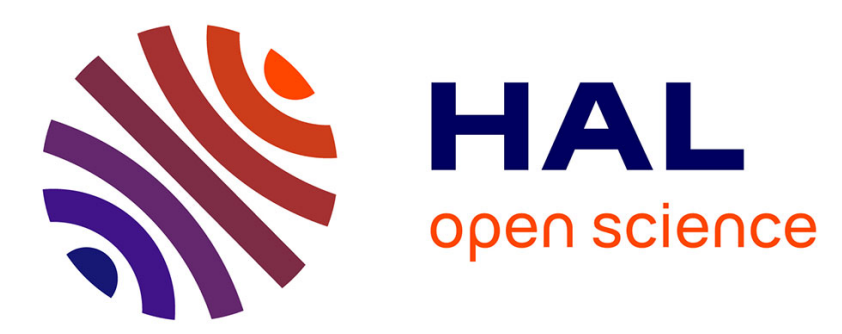

\title{
Dense omnidirectional RGB-D mapping of large scale outdoor environments for real-time localisation and autonomous navigation
}

\author{
Maxime Meilland, Andrew Ian Comport, Patrick Rives
}

\section{To cite this version:}

Maxime Meilland, Andrew Ian Comport, Patrick Rives. Dense omnidirectional RGB-D mapping of large scale outdoor environments for real-time localisation and autonomous navigation. Journal of Field Robotics, 2015, 32 (4), pp.474-503. 10.1002/rob.21531 . hal-01010429

HAL Id: hal-01010429

https://hal.inria.fr/hal-01010429

Submitted on 19 Jun 2014

HAL is a multi-disciplinary open access archive for the deposit and dissemination of scientific research documents, whether they are published or not. The documents may come from teaching and research institutions in France or abroad, or from public or private research centers.
L'archive ouverte pluridisciplinaire HAL, est destinée au dépôt et à la diffusion de documents scientifiques de niveau recherche, publiés ou non, émanant des établissements d'enseignement et de recherche français ou étrangers, des laboratoires publics ou privés. 


\title{
Dense omnidirectional RGB-D mapping of large scale outdoor environments for real-time localisation and autonomous navigation
}

\author{
Maxime Meilland \\ INRIA, I3S/CNRS \\ University of Nice Sophia Antipolis \\ maxime.meilland@i3s.unice.fr
}

\author{
Andrew I. Comport \\ I3S/CNRS \\ University of Nice Sophia Antipolis \\ andrew . comport@cnrs.fr
}

\author{
Patrick Rives \\ INRIA Sophia Antipolis \\ patrick.rives@inria.fr
}

\begin{abstract}
This paper presents a novel method and innovative apparatus for building 3D dense visual maps of large-scale unstructured environments for autonomous navigation and real-time localisation. The main contribution of the paper is focused on proposing an efficient and accurate 3D world representation that allows to extend the boundaries of state-of-the-art dense visual mapping to large-scales. This is achieved via an omni-directional key-frame representation of the environment which is able to synthesise photo-realistic views of captured environments at arbitrary locations. Locally the representation is image-based (ego-centric) and is composed of accurate augmented spherical panoramas combining photometric information (RGB), depth information (D) and saliency for all viewing directions at a particular point in space (i.e. a point in the light field). The spheres are related by a graph of 6 degrees of freedom poses (3 dof translation and 3 dof rotation) which are estimated through multi-view spherical registration. It is shown that this world representation can be used to perform robust real-time localisation (in 6 dof) of any configuration of visual sensors within their environment whether they be monocular, stereo or multi-view. Contrary to
\end{abstract}


feature-based approaches, an efficient direct image registration technique is formulated. This approach directly exploits the advantages of the spherical representation by minimising a photometric error between a current image and a reference sphere. Two novel multi-camera acquisition systems have been developed and calibrated to acquire this information and this paper reports for the first time the second system. Given the robustness and efficiency of this representation, field experiments demonstrating autonomous navigation and large-scale mapping will be reported in detail for challenging unstructured environments, containing vegetation, pedestrians, varying illumination conditions, trams and dense traffic.

\section{Introduction}

Autonomous navigation in complex and dynamic unstructured environments over large-scale distances, is an active field in robotics. One of the major difficulties in this objective is precise localisation of the vehicle within its environment so that autonomous navigation techniques can be employed. Indeed, robust localisation, particularly in heavily occluded areas (such as canyons, tunnels or forest areas), is a non-trivial problem due to GPS masking and poor precision of low cost units. Classical dead reckoning methods like odometry, typically performed by inertial sensors and wheels encoders are prone to drift and therefore are not suited to large distances.

Whilst very powerful platforms exist such as the Google car (Guizzo, 2011), they rely on a plethora of sensors which are fused together in a probabilistic framework. Perhaps the most important sensor is the 3D Velodyne Lidar which is an active sensor that is extremely expensive when compared to cheap off-the-shelf cameras. Relatively recent performance improvements in computing hardware have, however, made real-time computer vision suitable for autonomous navigation of mobile robots. Visual odometry, which estimates the full 6 degrees of freedom (dof) of vehicle motion from image sequences produces very precise results and has lower drift than the most expensive IMU's (Howard, 2008).

Visual odometry methods (Nistér et al., 2004; Comport et al., 2007; Konolige and Agrawal, 2008) are, however, incremental and prone to small drifts, which when integrated over time, become increasingly significant over large distances. In a similar way, simultaneous localisation and mapping approaches (Montemerlo et al., 2002; Davison and Murray, 2002; Thrun, 2002; Durrant-Whyte and Bailey, 2006; Klein and Murray, 2007) allow both localisation and cartography, giving 
alternative and promising solutions. Classically based on the Extended Kalman filter, these techniques have proven to work over very long trajectories, however, their robustness and accuracy is highly dependant on tuning low level feature extraction-matching and the filtering approach often smooths out important non-linear information.

A solution to minimise drift is to use a pre-computed 3D model of the environment obtained during a learning phase. This kind of approach has several advantages compared to pure SLAM approaches:

- Map building is performed via a learning phase which can be computed off-line using powerful yet computationally expensive techniques.

- More computational effort can be dedicated to robust real-time pose estimation online.

- Localisation with respect to the 3D model remains drift-free.

- If the $3 \mathrm{D}$ model is at scale, monocular localisation is at scale too.

In visual navigation, two main classes of approach exist, global parametric 3D models and image-based keyframe models. In the first case, 3D parametric models have been extensively used for object tracking by minimising the re-projection error between the model and salient features in the images (Marchand et al., 2001; Drummond et al., 2002; Vacchetti et al., 2004; Comport et al., 2006; Lothe et al., 2010). This kind of approach represents the 3D data in a single global reference frame and has difficulty in representing local data accurately. Even if automatic techniques which build 3D parametric models of large-scale environments are becoming more and more accurate (Hammoudi et al., 2010; Craciun et al., 2010; Lafarge and Mallet, 2011), they rely heavily on the structure of urban environments and are therefore not well adapted to unstructured environments.

On the other hand, key-frame techniques aim at representing the world with a set of images positioned in $2 \mathrm{D}$ or $3 \mathrm{D}$, without performing an explicit $3 \mathrm{D}$ reconstruction of the environment. This allows to store raw (unmodified) local sensor data in the representation for high accuracy and maintains a topological framework at large-scale that is accurate enough to ensure the connectivity between locally precise keyframes. In the literature, several approaches have successively performed localisation using an image-based memory. In (Royer et al., 2005; Konolige and Agrawal, 2008), a database of key-frames, containing geometric points features (Harris) is used for online camera localisation. A similar approach is proposed in (Courbon et al., 2009) with an omnidirectional camera. In (Jogan and Leonardis, 2000) a cylindrical panorama memory is proposed, and in (Cobzas et al., 2003) a panoramic image memory combined with depth information extracted from a laser scan is used for localisation. 
Those approaches, however, all rely on feature extraction and matching, and therefore do not take full advantage of the dense information contained in the images. Our main contribution is to propose an accurate and robust image-based representation which allows to map large-scale unstructured environments as densely as possible. The aim is to provide a world model that provides maximal robustness and maximal flexibility for online localisation and navigation purposes. Online real-time localisation efficiency is achieved, not by extracting and matching features, but by proposing a novel non-linear saliency measure for individual pixels. In particular, the proposed representation is composed of a graph of augmented visual spheres containing omnidirectional RGB-D information (colour and depth) for many spatial locations. This representation allows to efficiently perform online localisation and navigation using state of the art dense registration techniques (Comport et al., 2007; Meilland et al., 2010; Meilland et al., 2012).

This present publication is the culmination of many previously published articles which were carried out in the context of the French national CityVIP project aimed at autonomous vehicle navigation. The individual contributions have never been published together and the aim of this article is to provide the global scope of this complex system whilst demonstrating new experimental results for several large-scale field experiments:

- In (Comport et al., 2010) a dense direct approach was proposed for visual odometry.

- In (Meilland et al., 2010) a spherical representation was proposed along with a saliency selection approach.

- In (Meilland et al., 2011a) a spherical sensor and real-time localisation with respect to a spherical database was proposed.

- In (Meilland et al., 2011b) a robust approach to illumination variations was proposed.

- In (Meilland et al., 2012) autonomous navigation in challenging environments was demonstrated.

This paper has been structured in such a way that the relevant literature review is included at the beginning of each section. As such, in Section 2 the global non-linear optimisation criterion that is minimised will be introduced and formalised. In Section 3 a dense spherical key-frame model will be introduced to represent the 3D world. Section 4 will detail a novel spherical acquisition system that was built for the purpose of this project to acquire streams of photometric colour and depth panoramas at $45 \mathrm{~Hz}$. In Section 5 we will provide computational tools to allow real-time localisation of different types of cameras with respect to this world model. Finally, Section 6 will detail several field experiments. Initial results for the acquisition and mapping of large-scale environments will be given. Results from several sites including the XIIth district of Paris, INRIA Sophia-Antipolis and Place Jaude in Clermont-Ferrand will be given. In a last part a fully 
autonomous, vision-only navigation system will be demonstrated to run successfully in a highly dynamic unstructured environment involving pedestrians, trams, cars and varying illumination conditions.

\section{Visual localisation}

Feature-based methods (e.g. (Howard, 2008; Davison and Murray, 2002; Nistér et al., 2004; Mouragnon et al., 2006; Mei et al., 2010; Kitt et al., 2010; Tardif et al., 2010)) rely on an intermediary estimation process based on thresholding (Harris and Stephens, 1988; Lowe, 2004) before requiring matching between frames to recover camera motion. This feature extraction and matching process is often badly conditioned, noisy and not robust, and therefore it must rely on higher level robust estimation techniques and on filtering.

Direct approaches (image-based), however, do not rely on this feature extraction and matching process. The camera motion is directly estimated by minimising a non-linear intensity error between images, via a parametric warping function. In this way, the matching and the motion estimation are performed simultaneously at each step of the optimisation. Classically direct approaches have focused on region-of-interest tracking whether they be modelled by Affine (Hager and Belhumeur, 1998), planar (Lucas and Kanade, 1981; Irani and Anandan, 1998; Irani and Anandan, 2000; Baker and Matthews, 2001; Malis, 2004; Dame and Marchand, 2010), or multiple-plane tracking (Mei et al., 2006; Silveira et al., 2008; Shi and Tomasi, 1994; Caron et al., 2011). In (Comport et al., 2007) direct approaches were generalised to use the full-image densely and track 6 dof pose using stereo cameras whilst mapping the environment through dense stereo matching. This approach allowed direct methods to perform accurate and robust visual odometry over large-scales.

\subsection{Direct $3 \mathrm{D}$ registration}

To begin, consider an image $\mathbf{I}$ with a colour brightness function $\mathbf{I}: \Omega \rightarrow \mathbb{R}^{+} ;(\mathbf{p}) \mapsto \mathbf{I}(\mathbf{p})$, where $\Omega=$ $[1, n] \times[1, m] \subset \mathbb{R}^{2}, \mathbf{P}=\left(\mathbf{p}_{1}, \mathbf{p}_{2}, \ldots, \mathbf{p}_{n m}\right)^{\top} \in \mathbb{R}^{m n \times 2} \subset \Omega$ are pixel locations within the image, and $n \times m$ is the dimension of the image. Now consider that for each pixel of the image, a depth information is also available such that $\mathbf{Z}: \Omega \rightarrow \mathbb{R}^{+} ;(\mathbf{p}) \mapsto \mathbf{Z}(\mathbf{p})$. It is convenient to consider the set of measurements in vector form such that $\mathbf{I}(\mathbf{P}) \in \mathbb{R}^{\mathrm{nm} \times 1}$ and $\mathbf{Z}(\mathbf{P}) \in \mathbb{R}^{\mathrm{nm} \times 1}$. The set $\mathcal{I}=\{\mathbf{I}, \mathbf{Z}\}$ will be denoted as an augmented image. 
The objective here is to register a current image $\mathbf{I}$ with a reference augmented image $\mathcal{I}^{*}$, where $\mathbf{I}$ is undergoing a full 3D transformation $\widetilde{\mathbf{T}}(\mathbf{x})=(\mathbf{R}, \mathbf{t}) \in \mathbb{S E}(3)$ defined between $\mathbf{I}$ and $\mathbf{I}^{*}$. A superscript $*$ will be used throughout to designate the reference view variables.

Throughout, $\mathbf{R} \in \mathbb{S O}(3)$ is a rotation matrix and $\mathbf{t} \in \mathbb{R}(3)$ a translation vector. The vector $\mathbf{x}=(\boldsymbol{\omega}, \boldsymbol{v}) \in \mathbb{R}^{6}$ is the 6 degrees of freedom twist, which is related to a pose $\mathbf{T}$ via the matrix exponential:

$$
\mathbf{T}(\mathbf{x})=e^{[\mathbf{x}]_{\wedge}}=\int_{0}^{1} \mathbf{x} \mathrm{d} t \in \mathbb{S E}(3)
$$

with the operator $[.]_{\wedge}$ as:

$$
[\mathbf{x}]_{\wedge}=\left[\begin{array}{cc}
{[\boldsymbol{\omega}]_{\times}} & \boldsymbol{v} \\
\mathbf{0} & \mathbf{0}
\end{array}\right] \in \mathfrak{s e}(3)
$$

where $[\cdot]_{\times}$represents the skew symmetric matrix operator.

Under full brightness consistency assumption, the current image intensities can be warped via novel view synthesis (Avidan and Shashua, 1997) onto the reference view such that

$$
\mathbf{I}^{*}\left(\mathbf{P}^{*}\right)=\mathbf{I}\left(w\left(\mathbf{T} ; \mathbf{Z}^{*}, \mathbf{P}^{*}\right)\right)
$$

where the warping function $w(\cdot)$ transfers the current image intensities onto the reference pixels $\mathbf{P}^{*}$ via the depth-map $\mathbf{Z}^{*}$. This warping function depends on the acquisition sensor, and can be for example a perspective projection, an omnidirectional projection or a spherical projection (see Appendix (9.1)).

Now supposing that only a close approximation $\widehat{\mathbf{T}}$ of the true pose $\widetilde{\mathbf{T}}$ is available. The aim is to estimate the incremental pose transformation $\mathbf{x}$ of the true value $\widetilde{\mathbf{x}}$ which satisfies

$$
\widetilde{\mathbf{T}}=\widehat{\mathbf{T}} \mathbf{T}(\mathbf{x})
$$

The unknown $\mathbf{x}$ can be estimated by minimising the following intensities error:

$$
\mathbf{e}(\mathbf{x})=\rho\left(\mathbf{I}\left(w\left(\widehat{\mathbf{T}} \mathbf{T}(\mathbf{x}) ; \mathbf{Z}^{*}, \mathbf{P}^{*}\right)\right)-\mathbf{I}^{*}\left(\mathbf{P}^{*}\right)\right)
$$

where $\rho(\cdot)$ is a robust outlier rejection computed by M-estimation using Huber influence function (Huber, 1981). This function allows to rejects outliers such as moving objects and local illumination changes between the images. 


\section{$2.2 \quad$ Non-linear minimisation}

The error function of equation (4) can be minimised using an iteratively re-weighted least squared optimisation:

$$
\mathbf{x}=\underset{\mathbf{x}}{\arg \min } \mathbf{e}(\mathbf{x})^{\top} \mathbf{D e}(\mathbf{x})
$$

by $\left.\nabla \mathbf{e}(\mathbf{x})\right|_{\mathbf{x}=\tilde{\mathbf{x}}}=\mathbf{0}$, where $\nabla$ is the gradient operator with respect to the unknown $\mathbf{x}$, assuming a global minimum is reached at $\mathbf{x}=\tilde{\mathbf{x}}$.

An efficient second order minimisation (ESM) is employed (Malis, 2004), which allows to pre-compute most of the minimisation parts directly on the reference image. In this case the unknown $\mathbf{x}$ is iteratively updated using a Gauss-Newton like procedure:

$$
\mathbf{x}=-\left(\mathbf{J}^{\top} \mathbf{D J}\right)^{-1} \mathbf{J}^{\top} \mathbf{D e}
$$

where $\mathbf{Q}=\mathbf{J}^{T} \mathbf{D} \mathbf{J}$ is the robust Gauss-Newton Hessian approximation. $\mathbf{J}$ is the warping Jacobian matrix of dimension $n m \times 6$. $\mathbf{D}$ is a diagonal weighting matrix of dimensions $n m \times n m$ related to the robust function $\rho(\cdot)$ (see details in Appendix (9.3)).

The pose estimate is updated at each step by an homogeneous transformation:

$$
\widehat{\mathbf{T}} \leftarrow \widehat{\mathbf{T}} \mathbf{T}(\mathbf{x})
$$

and the minimisation is iterated until the increments on $\mathbf{x}$ are sufficiently small.

\subsection{Multiple key-frame registration}

In Section 2.1, only one reference image was considered. This approach is highly incremental and prone to drift and linearisation approximation. If more than one reference image is available then it is possible to consider global bundle adjustment approaches (Triggs et al., 2000), however, these approaches are not computationally efficient for real-time applications. Furthermore, in large scale mapping approaches, the connectivity between the various camera poses is relatively sparse meaning that performing global bundle adjustment is not efficient. Alternatively, it is possible to consider sliding window bundle adjustment (Mouragnon et al., 2006; Sibley et al., 2008), which improves performance at little cost. 
In this paper, the localisation problem will be formulated as a simultaneous minimisation of several intensities from $N$ locally close reference images from a key-frame graph such that

$$
\mathbf{e}(\mathbf{x})=\left[\begin{array}{c}
\left.\mathbf{I}\left(w\left(\widehat{\mathbf{T}} \mathbf{T}(\mathbf{x}) \mathbf{I} ; \mathbf{Z}^{1}, \mathbf{P}^{1}\right)\right)-\mathbf{I}^{1}\left(\mathbf{P}^{1}\right)\right) \\
\vdots \\
\left.\mathbf{I}\left(w\left(\widehat{\mathbf{T}} \mathbf{T}(\mathbf{x}) \mathbf{T}^{N} ; \mathbf{Z}^{N}, \mathbf{P}^{N}\right)\right)-\mathbf{I}^{N}\left(\mathbf{P}^{N}\right)\right)
\end{array}\right],
$$

where $\mathbf{T}^{N}$ is the transformation between each key-frame.

\subsection{Multi-resolution approach}

One of the major drawbacks of direct approaches, and more generally of iterative approaches, is that the

initial pose $\widehat{\mathbf{T}}$ must be close to the solution $\widetilde{\mathbf{T}}$ to converge. The convergence domain and the algorithm performance can be improved using a coarse-to-fine optimisation strategy. This is achieved using multi-resolution image pyramids (e.g. constructed by Gaussian filtering and sub-sampling (Burt and Adelson, 1983)). The minimisation begins at the lowest resolution and the result is used to initialise the next level repeatedly until the highest resolution is reached. This greatly improves the convergence domain/speed and some local minima can be avoided.

\section{3D world representation}

\subsection{Parametric models}

Many approaches in the literature use parametric models to efficiently represent the environment and store prior information about the scene. CAD models can be used to directly inject prior information about the 3D model into a localisation system and some examples include (Brown, 1971; Lowe, 1991; Marchand et al., 2001; Drummond et al., 2002; Vacchetti et al., 2004; Comport et al., 2006). In this case camera pose (orientation and translation) is estimated with respect to the model reference frame by minimising an error between the re-projected 3D model and corresponding features extracted from the images. The features may include points, lines (Lowe, 1991; Marchand et al., 2001), planes (Benhimane and Malis, 2004), contours (Drummond et al., 2002), cylinders or even combinations of them (Comport et al., 2006). Whilst these algorithms work well, they are based on highly complicated modelling procedures as well as structured 
visual features in the images, meaning that they are rarely used for environments larger than the scale of a room and they do not easily scale to unstructured environments.

Several studies have been conducted to improve localisation techniques for larger scale environments using parametric models. (Lothe et al., 2010) uses an approximate global 3D model to align a local map, reconstructed using a visual SLAM approach, with the geometric part of the model in order to correct for drift. In (Cappelle et al., 2011), a 3D model is only used to detect obstacles observed between the images perceived by a camera and virtual images whilst the localisation of the camera is obtained by GPS-RTK. In (Irschara et al., 2009) a sparse 3D point model is reconstructed using a $S f M$ algorithm and is used to locate a camera through matched SIFT points.

In general, these parametric models are capable of representing environments or target objects in an efficient yet approximate way. Even if methods for automatically reconstructing large-scale environments using parametric models are becoming more and more precise (Hammoudi et al., 2010; Craciun et al., 2010; Furukawa and Ponce, 2010; Lafarge and Mallet, 2011; Shan et al., 2013), the resulting reconstructed models remain not completely photo-realistic and cannot easily be compared directly with live sensor data. This type of representation is heavily influenced by 3D modelling tools provided by the computer graphics community who are only interested in rendering visual pleasing scenes but not in representing the sensor data as best as possible. Realistic computer graphics models are obtained by texture mapping onto 3D planar façades which have been extracted from the environment. Unfortunately this type of approximation introduces many artefacts and visual inconsistencies which cannot easily be overcome by robust estimation methods. To be robust to these errors, (?) proposes to use a mutual information approach (Viola and Wells, 1995) in order to register a synthetic image generated from an approximate textured 3D model with a real image. This metric allows to compare highly different images (between the sensor and model), however the calculations required for the alignment are computationally expensive.

\section{$3.2 \quad$ Image-based models}

An alternative approach to using parametric 3D models based on features is to represent the environment using an image-based approach also known as an ego-centric model. In this case key-frame images, acquired during a learning phase, are stored in a database and the sensor data remains in its raw un-modified form. The learning phase also allows to estimate the transformations between the key-frames and store their position in global world coordinates. The local information contained in the key-frames is then used to estimate the position of a camera navigating within the pre-mapped environment. Unlike the parametric 
methods, these maps are much simpler to acquire and they provide greater accuracy and robustness since the raw sensor data has not been approximated and a direct image error can be minimised. Furthermore, the topological connections between the key-frames avoid the necessity to represent all the local map data in a global reference frame and prevent introducing bias due to the choice of the world coordinate system. In this case the local sensor data remains accurate and is not contaminated by the global drift in the sensor pose.

In the literature, the proposed quantitative localisation methods using key-frames all rely on featurebased techniques ((Royer et al., 2005; Courbon et al., 2008)). In order to provide a maximum field of view, which better constraints 6 degrees of freedom pose estimation (Baker et al., 2001), a spherical representation is proposed. This kind of representation, already found in commercial applications such as Google Earth (cf. (Anguelov et al., 2010)), allows an immersive visualisation of the scene using panoramic images, but is not adapted for accurate localisation or navigation since the spherical images are very sparsely positioned in the world and accurate dense 3D maps are not available, even thought some recent work (Klingner et al., 2013) shows that SFM can be used to reconstruct dense point clouds and improve the images locations.

\subsection{Local dense representation: augmented visual sphere}

In the representation proposed in this paper, a local area in space is defined by an augmented spherical key-frame which captures the light field of all viewing directions from a particular point in 3D space along with its depth map. This allows to extend robust and accurate state-of-the-art dense full-image approaches (Comport et al., 2007) to its most general form (i.e. all viewing directions are densely modelled). To formalise this, each sphere will be defined by the set

$$
\mathcal{S}=\left\{\mathbf{I}_{S}, \mathbf{P}_{S}, \mathbf{Z}_{S}, \mathbf{W}_{S}\right\}
$$

where:

- $\mathbf{I}_{S}$ is the photometric spherical image. This image is obtained from the custom camera system presented in Section 4.3 by warping multiple images onto the sphere.

- $\mathbf{P}_{S}=\left\{\mathbf{q}_{1}, \ldots, \mathbf{q}_{n}\right\}$ is a set of evenly spaced points on the unit sphere where $\mathbf{q} \in S^{2}$. These points have been sampled uniformly on the sphere as in (Meilland et al., 2010). 

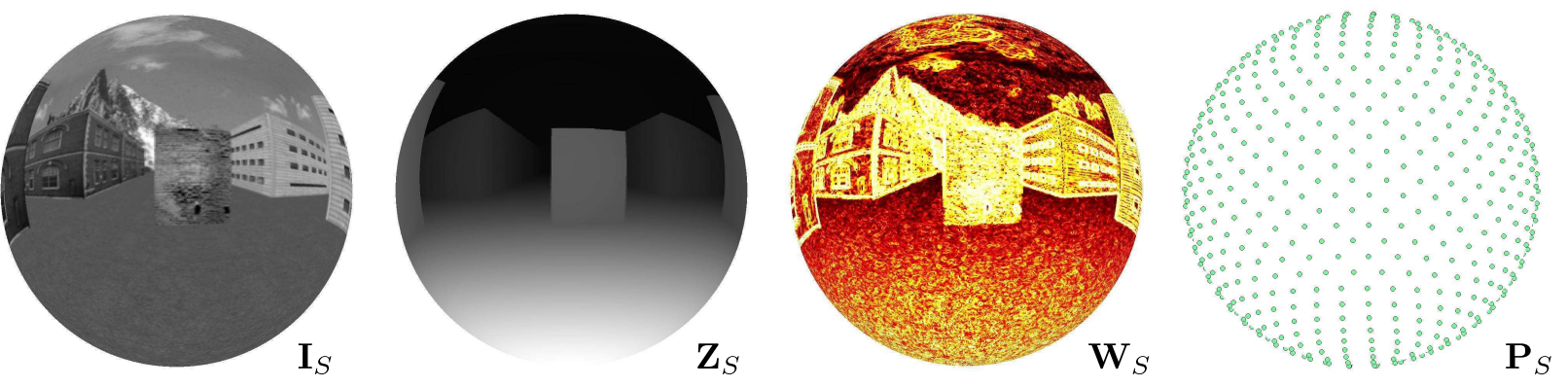

Figure 1: Local representation: augmented sphere $\mathbf{S}$ containing intensities $\mathbf{I}_{S}$, depth-map $\mathbf{Z}_{S}$, saliency $\mathbf{W}_{S}$ and a sampling $\mathbf{P}_{S}$.

- $\mathbf{Z}_{S}$ are the depths associated with each pixel which have been obtained from dense stereo matching. The $3 \mathrm{D}$ point is subsequently defined in the sphere as $\mathbf{V}_{S}=\left(\mathbf{P}_{S}, \mathbf{Z}_{S}\right)$.

- $\mathbf{W}_{S}$ is a saliency image which contains knowledge of good pixels to use for tracking applications. It is obtained using a non-linear pixel selection, computed by analysing the Jacobian of the warping function as detailed in Section 3.6.

\subsection{Global topologic representation: omnidirectional key-frame graph}

At global scale, each local spherical key-frame will be connected by a topological graph of poses. This ego-centric 3D model of the environment is defined by the following graph

$$
\mathcal{G}=\left\{\mathcal{S}_{1}, \ldots, \mathcal{S}_{n} ; \mathbf{x}_{1}, \ldots, \mathbf{x}_{m}\right\}
$$

where $\mathcal{S}_{i}$ are augmented spheres that are connected by a minimal parametrisation $\mathbf{x}$ of each pose.

The main advantages of this spherical representation are:

- An omnidirectional key-frame representation allows to conserve local sensor data (without transformation) and subsequently ensure photometric consistency. This enhances the performance of direct registration techniques (accuracy, robustness, convergence speed and domain of convergence).

- A topological graph representation encodes the viewing trajectory which allows to retain the uncertainty associated with the sensor measurements, yet a 3D model can be generated from this representation if needed. 


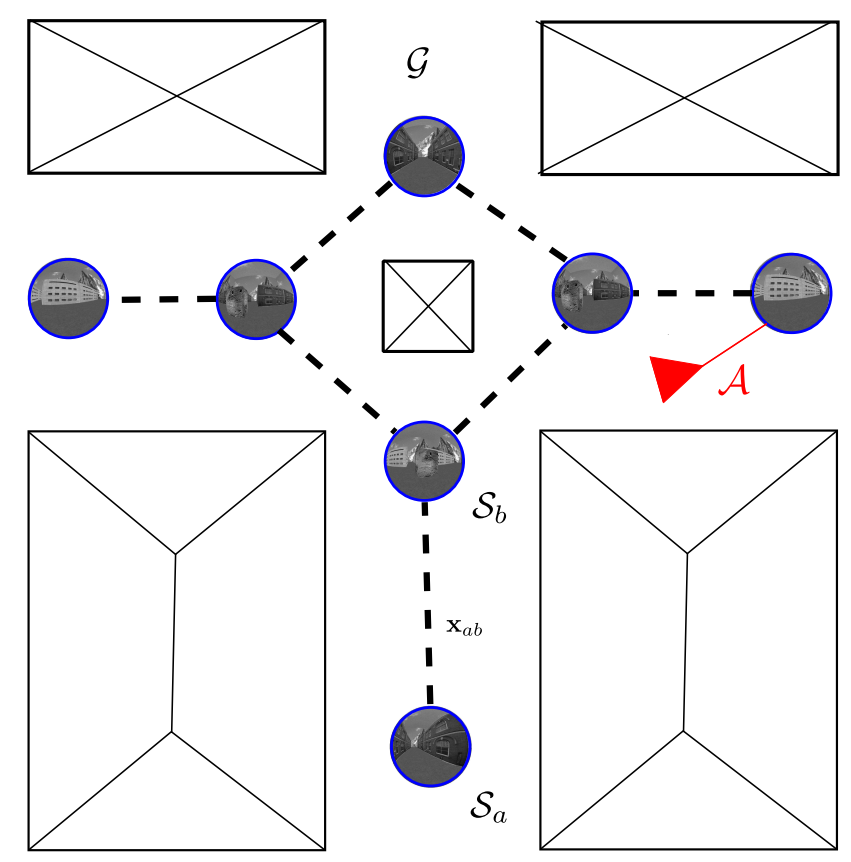

Figure 2: Ego-centric representation: graph of spheres $\mathcal{G}$ allowing the localisation of a vehicle $\mathcal{V}$ navigating locally within the graph.

- The map can be accessed in constant time independently of the graph size.

- Augmenting photometric spherical panoramas with dense depth allows to perform local novel view synthesis necessary for direct registration techniques.

- A spherical representation provides all local view directions and therefore provides a general representation that can encode data from different kinds of sensors like perspective cameras, multi-view cameras or omnidirectional cameras and laser range finders.

- Full-view sensors condition the observability of 3D motion well (Baker et al., 2001) which greatly improves robustness.

- This representation can be made invariant to illumination variation as in (Meilland et al., 2011b).

\subsection{Global spherical key-frame positioning}

To accurately recover the position of the spheres of the graph with respect to one-another, a 6 degrees of freedom spherical localisation model is used based on an accurate dense localisation (Comport et al., 2010)(Meilland et al., 2010). Considering $\mathcal{S}^{*}$, an augmented sphere defined in Section 3.3, the objective is to compute the pose between a reference sphere $\mathcal{S}^{*}$ and the next one $\mathcal{S}$. The 
localisation problem is solved using a dense visual odometry approach which uses a direct 3D image registration technique that provides accurate pose estimation. Since this is a local optimisation approach it is assumed that the camera frame-rate is high $(30 \mathrm{~Hz})$ and that inter-frame displacements are small.

The intensity error measure between a reference sphere and a current sphere is then defined as follows:

$$
\mathbf{e}(\mathbf{x})=\rho\left(\mathbf{I}_{s}\left(w\left(\widehat{\mathbf{T}} \mathbf{T}(\mathbf{x}) ; \mathbf{Z}_{S}^{*}, \mathbf{P}_{S}^{*}\right)\right)-\mathbf{I}_{s}^{*}\left(\mathbf{P}_{S}^{*}\right)\right)
$$

where $w($.$) is the spherical warping function which transfers 3D points onto the current sphere. The direct$ minimisation procedure was given in Section 2. The main difference with respect to equation (4) being that the sphere is sampled, uniformly if possible, within polar spherical coordinates. The interested reader can refer to this paper (Meilland et al., 2010) where the HealPix algorithm (Gorski et al., 2005) is used for uniform sampling.

\subsubsection{Spherical node selection}

Indeed, the vertices of the graph should be carefully placed in the world so as to represent the environment with little redundancy. One preliminary technique to achieve this goal locally is to observe criteria between an initially selected reference sphere and surrounding spheres. In practice, the trajectory of the acquisition system along a sequence is computed by integrating elementary displacements estimated from successive spherical registration. The strategy used here is to maintain as long as possible the reference sphere to minimise the drift introduced when a new reference sphere is taken. Therefore a new reference sphere is placed according to the Median Absolute Deviation (MAD):

$$
\lambda<\operatorname{Median}(|\mathbf{e}-\operatorname{Median}(\mathbf{e})|),
$$

where $\mathbf{e}$ is the vector of intensities error between the current sphere and the previous set of reference spheres defined in (11) at the end of the minimisation.

This criterion is directly related to the geometry of the environment via the warping function of equation (11), providing a robust measure of the photometric changes between the images, allowing to quantify:

- The amount of occlusions related to the scene geometry and the sensors viewpoints.

- The effective resolution changes related to the distance between the images. 


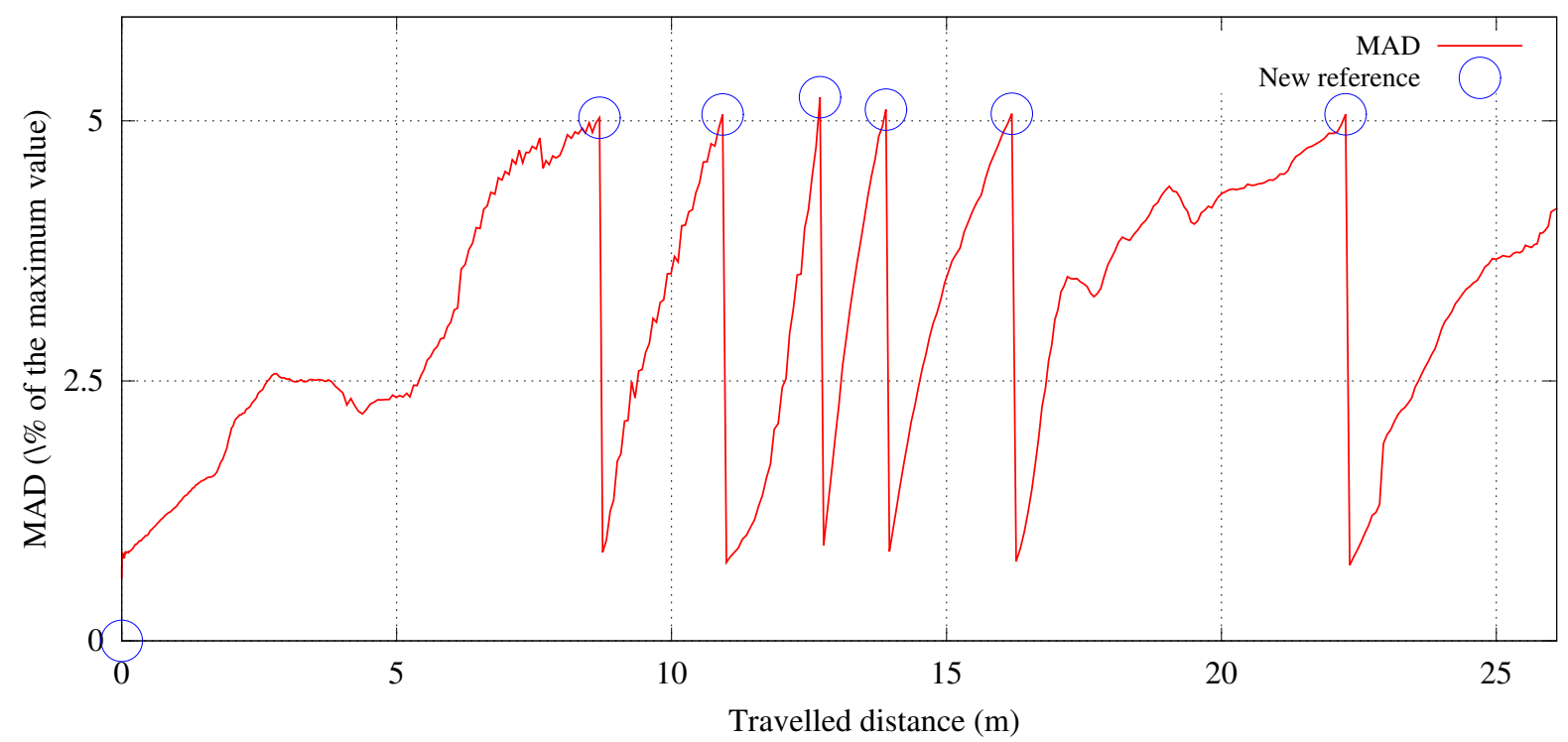

Figure 3: Evolution of the median absolute deviation (MAD) with respect to the travelled distance. The MAD value increases differently with respect to the scene configuration: between $0<\Delta<8$ and $16<\Delta<25$ the scene geometry is far from the observation viewpoint. Between $8<\Delta<16$ the scene geometry is close to the sensor. A new reference is reconstructed when the MAD exceed $5 \%$ of the maximum intensity error (e.g. 255 grey levels).

Figure 3, shows an example of the evolution of the median absolute deviation for a 500 images trajectory with respect to the distance $\Delta$ travelled by the vehicle. When the environment is far from the camera viewpoint $(0<\Delta<8$ and $16<\Delta<25)$, the MAD increases slowly, due to very small changes between the images. When the environment is closer to the camera $(8<\Delta<16)$, the MAD value increases rapidly until the selection threshold $\lambda$ is reached, which allows to sample more spheres only when necessary.

\subsubsection{Loop closure detection and drift compensation}

Since a dense spherical visual odometry technique was used, small amounts of drift are integrated along the sequence (typically $1 \%$ which could cause the final graph to become inconsistent or redundant (i.e. a route mapped twice in opposite directions). To correct the drift and remove redundant spheres, the spherical loop closing technique proposed by (Chapoulie et al., 2011) was used. This method is based on SIFT descriptors augmented by their distribution across the sphere which is represented by histograms. A dictionary is incrementally built online which allows to detects images of similar appearance, regardless of the image orientation. This approach was specifically designed for the spherical key-frame representation proposed in this paper. 
When a loop closure is detected, a dense registration is performed between the candidates and the detection is validated if the residual error after the minimisation is below a pre-defined threshold. Using the new pose provided by the loop closure, the graph is augmented with the new constraints and then, optimised using the pose-graph library TORO (Grisetti et al., 2009). This open-source library implements the method given

in (Grisetti et al., 2007) which is an extension of (Olson et al., 2006). It allows the errors introduced by the new constraints in the graph to be minimised by a stochastic gradient descent method.

Figure 4 shows the benefits of using the loop closures. Top image corresponds to the estimated trajectory done with only visual odometry. The start point and the end point should be at the same position. Middle image corresponds to the corrected trajectory taking into account loop closures. Bottom image details a place where loop closures are detected from perpendicular points of view.

Beside of compensating the drift, the loop closure detection method allows us to drastically reduce the size of the graph by merging the redundant spherical views.

\subsection{Information selection}

The essence of direct approaches is to minimise pixel intensities directly between images. Images are, however, clearly redundant, i.e. a lot of information is not overly important for navigation, such as completely untextured parts. This kind of information should not be used for several reasons. One of them is that if spheres are constructed from a panoramic multi-camera system at high resolution, the number of pixels to warp at each iteration of the real-time minimisation process could be extremely large. Therefore reducing the dimension is essential for real-time computing.

A classic approach (Baker and Matthews, 2001; Comport et al., 2007) often employed with direct techniques, is to sort and select only the best corners or edges (intensity gradients) in grey-level images:

$$
i=\underset{i}{\arg \max }\|\nabla \mathbf{I}(i)\|
$$

This naive approach does not consider the importance of the structure of the scene and can lead to the selection of non-observable measurements. For example, selecting high intensity gradient points at infinity, rather than more informative but low gradient close points in a scene, will yield imprecise results. In the worst case it will not even be possible to estimate translation because of the invariance of infinite points in pure translation. At the same time infinite points are also useful because they improve the stability and robustness 

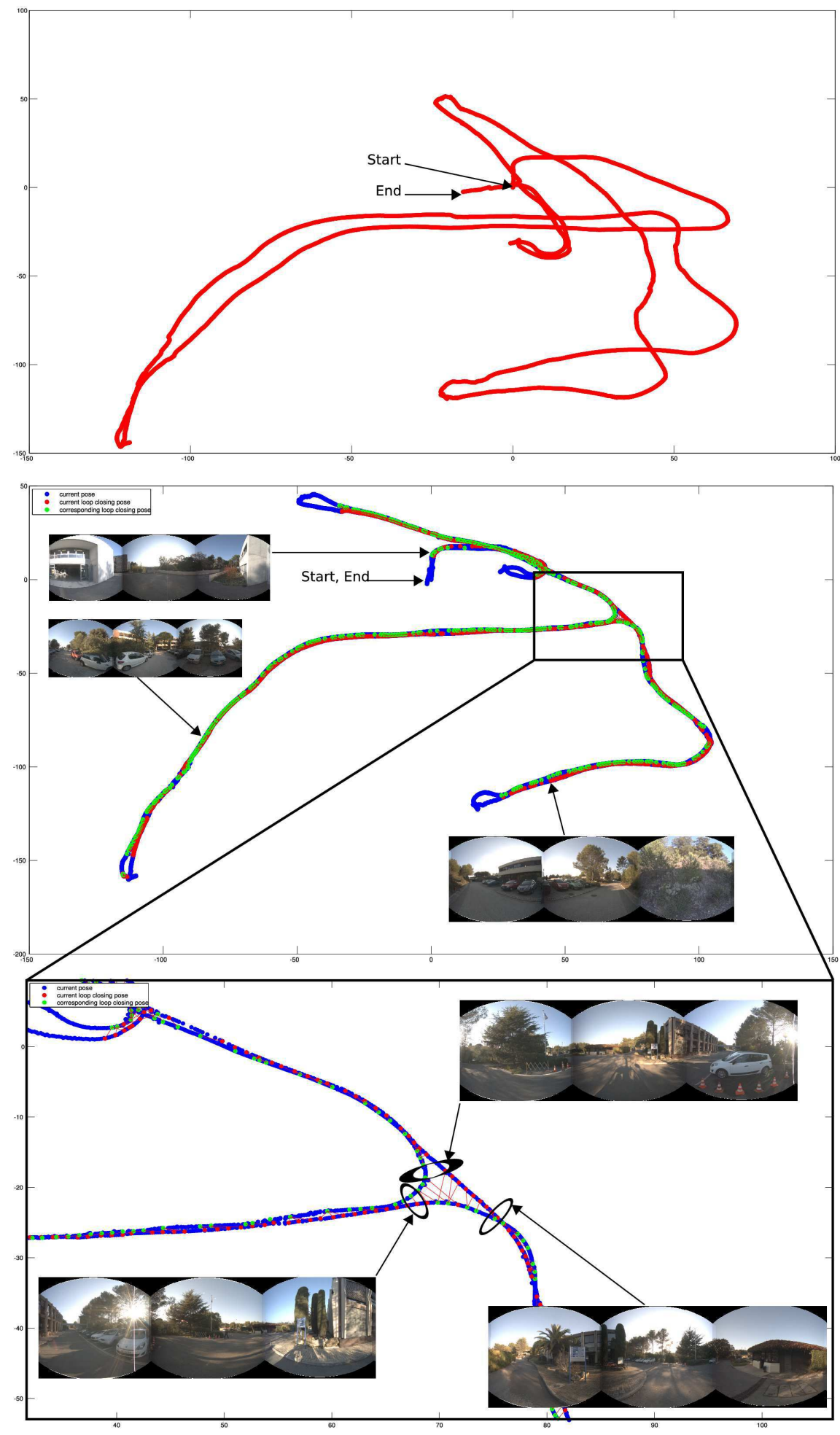

Figure 4: Drift correction using loop closures: Top image corresponds to the estimated trajectory done with only visual odometry. The start point and the end point should be at the same position. Middle image corresponds to the corrected trajectory taking into account loop closures. Bottom image details a place where loop closures are detected from perpendicular points of view. 
of the tracking algorithm due to their small displacement in the images for large translations. The difficulty is therefore to analyse the accuracy and robustness of selected points. In (Dellaert and Collins, 1999), a saliency map is build by sorting the pixels with respect to the variance of their Jacobian. A subset is then randomly extracted from the best pixels. This approach is finally applied to estimate only rotations. In (Benhimane et al., 2007), only linear or quadratic subsets are extracted for template-based tracking. Such subsets are good for linear convergence allowing to converge quickly. Unfortunately, this method suggests rejecting strong edges which are essential for obtaining good precision.

The novelty of the approach proposed here is to quantify the effect of the geometric structure and the image intensity measurements on navigation by analysing directly the entire analytical Jacobian which relates scene movement to sensor movement. The aim being to select points which best condition the six degrees of freedom of the pose. Indeed, the reference Jacobian Matrix $\mathbf{J}(\tilde{\mathbf{x}})$ directly combines grey level gradient (image derivatives) and geometric gradient such that $\mathbf{J}(\tilde{\mathbf{x}})=\mathbf{J}_{\mathcal{I}^{*}} \mathbf{J}_{G}$. This matrix can be decomposed into six parts corresponding to each degree of freedom of the transformation (called steepest descent images by (Baker and Matthews, 2001)):

$$
\mathbf{J}(\tilde{\mathbf{x}})=\left[\begin{array}{llllll}
\mathbf{J}^{1} & \mathbf{J}^{2} & \mathbf{J}^{3} & \mathbf{J}^{4} & \mathbf{J}^{5} & \mathbf{J}^{6}
\end{array}\right] \in \mathbb{R}^{n m \times 6} .
$$

Each column vector of $\mathbf{J}$ contains the gradient associated to one degree of freedom, and can be interpreted as a $m \times n$ saliency map after re-ordering its elements into a matrix. Figure 5 shows an example of 6 saliency maps, where a bright value indicates a strong gradient. The first 3 images $\left(\left|\mathbf{J}^{1}\right|,\left|\mathbf{J}^{2}\right|\right.$ et $\left.\left|\mathbf{J}^{3}\right|\right)$ show the translational part: close 3D measurements in the scene have a strong gradient. The last 3 images $\left(\left|\mathbf{J}^{4}\right|,\left|\mathbf{J}^{5}\right|\right.$ et $\left|\mathbf{J}^{6}\right|$ ) show the rotational components, which are invariant to the scene geometry.

The objective here is to extract a subset $\overline{\mathbf{J}}=\left\{\overline{\mathbf{J}}^{1}, \overline{\mathbf{J}}^{2}, \overline{\mathbf{J}}^{3}, \overline{\mathbf{J}}^{4}, \overline{\mathbf{J}}^{5}, \overline{\mathbf{J}}^{6}\right\} \subset \mathbf{J}$, of dimensions $p \times 6$, with $p \ll n m$, containing the pixels which best condition each degree of freedom of $\mathbf{J}$.

The proposed approach is a sorting algorithm, where each line $k$ of the new matrix $\overline{\mathbf{J}}$ is obtained by:

$$
\overline{\mathbf{J}}_{k}=\underset{j}{\arg \max }\left(\left|\mathbf{J}_{i}^{j}\right| \backslash \tilde{\mathbf{J}}\right),
$$

which correspond to select an entire line of the original matrix $\mathbf{J}$, corresponding to best gradient of the $j^{\text {th }}$ column. $\tilde{\mathbf{J}} \subset \overline{\mathbf{J}}$ is an intermediate subset containing the lines of $\mathbf{J}$ that are already selected, and $\backslash \tilde{\mathbf{J}}$ avoids to 


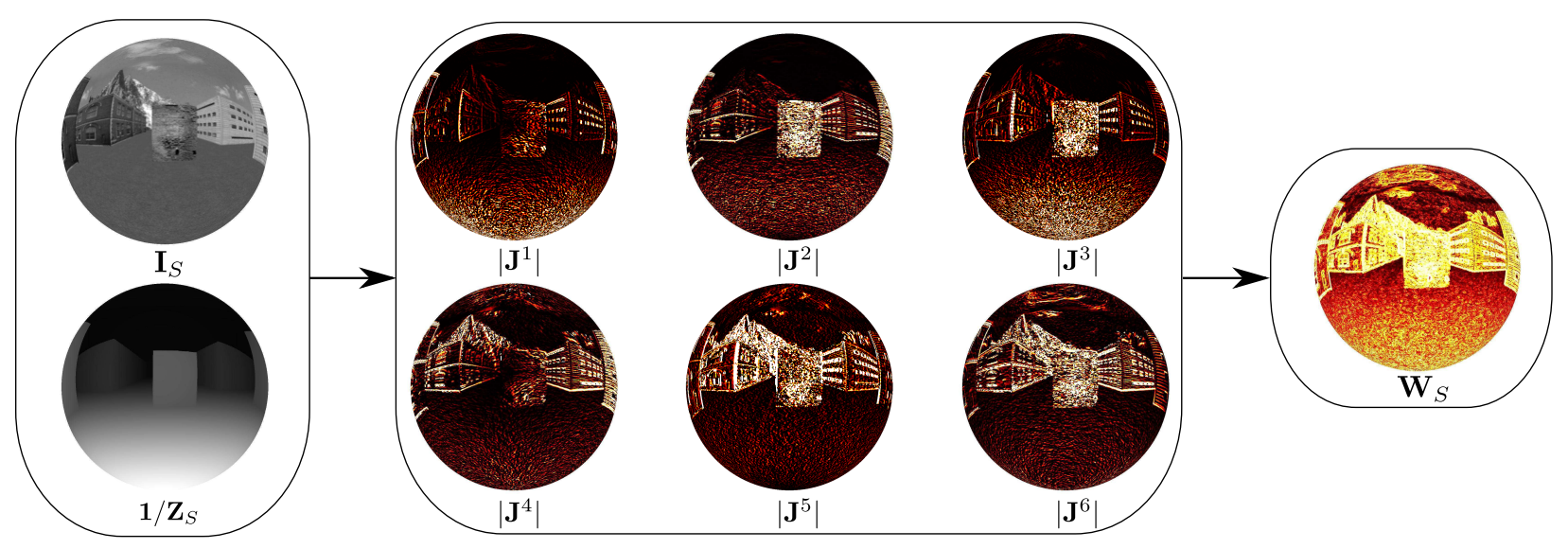

Figure 5: $\mathbf{I}_{S}$ : Reference image. $\mathbf{Z}_{S}$ : Depth-map. $\left|\mathbf{J}^{i}\right|$ : absolute value of the re-order Jacobian matrix for each degree of freedom. $\mathbf{W}_{S}$ : final saliency map after selection (first selected pixels are bright, last pixels are dark). From the 6 saliency maps, corresponding to the 6 columns of the Jacobian matrix, the proposed algorithm iteratively selects the bests pixels to produce a final saliency map containing the sorted values, which maximises the observability of each degree of freedom.

select the same line twice. The term (15) is iteratively applied for each degree of freedom, allowing to select the same amount of pixel for each degree of freedom, until all pixels are sorted.

In practice, the matrix $\overline{\mathbf{J}}$ is not explicitly reconstructed ( $c f$. algorithm 1 ), the indices of each line of $\mathbf{J}$ are stored in saliency order in the saliency map $\mathbf{W}_{S}$. During the learning phase, $\mathbf{J}(\tilde{\mathbf{x}})$ is pre-computed for each reference image and the entire pixels are sorted according to (15). During the online localisation phase, a dynamic selection of the pixel is performed with respect to the saliency map and the current camera viewpoint, allowing to use only the best $p$ pixels that are visible by the camera, whilst maximising the observability of the 6 degrees of freedom. In the next warping functions, this dynamic selection will be denoted by the function $s(\cdot)$ :

$$
\left(\mathbf{Z}^{s}, \mathbf{P}^{s}\right)=s(\mathbf{Z}, \mathbf{P})
$$

which return the best subset of pixels from the saliency map $\mathbf{W}_{S}$.

Since a multi-resolution approach is employed for the registration, the selection algorithm is performed on each resolution of the reference image and stored in the database.

\section{Spherical sensors}

The spherical key-frame model presented in the previous section requires an adequate system for acquiring these types of measurements. Due to the novelty of this representation, however, no classical sensors were 


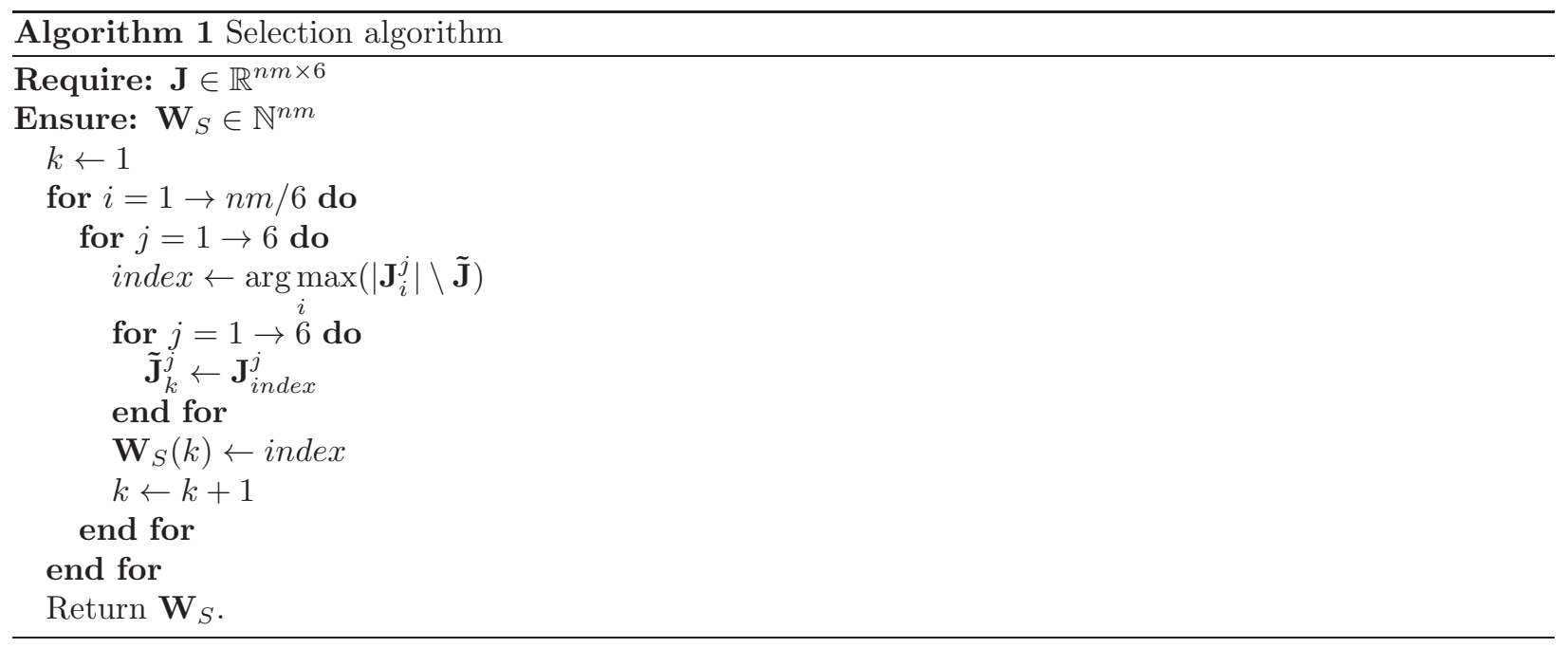

available and it was necessary to develop a customised system. As such, several classic omnidirectional and panoramic camera systems are analysed for this purpose and two new multi-view RGB-D camera system are presented.

\subsection{Photometric sphere}

Capturing omnidirectional views of the environment can be achieved using either omnidirectional catadioptric cameras (Nayar, 1997) or with multiple perspective cameras. An omnidirectional camera allows to capture panoramic images with a $360^{\circ}$ horizontal field of view. It, however, suffers from a low and non-uniform spatial resolution, and a limited vertical field of view (half-sphere, $<90^{\circ}$ ). This kind of sensor is therefore not efficient for outdoor mapping. Multi-view stitching techniques allow to build high resolution panoramic images by stitching and blending perspective images together (Szeliski, 2006). Those images can be acquired using several cameras (Baker et al., 2001), or from a sequence of images (Lovegrove and Davison, 2010). This kind of approaches, always assumes a unique centre of projection and may suffer from parallax artefacts.

\subsection{Depth sphere acquisition}

Passive systems are capable of simultaneously capturing a dense depth-map from cameras alone without any additional system. To our knowledge only omnidirectional stereo viewing systems have been developed and studied and they include (Lui and Jarvis, 2010; Micusik and Pajdla, 2004; Goncalves and Araujo, 2004; Caron et al., 2011; Arican and Frossard, 2007; He et al., 2007; Ragot et al., 2008). Multi-view spherical systems have been also widely studied in the literature, however, it is generally assumed that all cameras view 
the same object and that the cameras are all converging to that point (Kutulakos and Seitz, 2000). On the other hand, diverging multi-camera systems (Baker et al., 2001; Szeliski, 2006) all aim to have a common centre of projection and are unable to compute a depth image.

Active systems require projecting laser or light patterns onto the environment to obtain dense depth measurements. In (Gallegos et al., 2010), a spherical image is obtained using a classic omnidirectional camera. The depth information is obtained using a plan laser range finder (LRF) aligned with the camera optical centre. This approach assumes a planar and structured environment to propagate the depth onto the sphere. In (Cobzas et al., 2003) a similar idea is used but a pan/tilt perspective camera is used with a Lidar to reconstruct cylindrical augmented panoramas. Active RGB-D sensors such as Microsoft Kinect and Asus Xtion are actually popular in the robotics community. The projection of an infra-red pattern allows to recover dense depth-maps in real-time and can be used in SLAM systems such as (Audras et al., 2011; Newcombe et al., 2011a; Henry et al., 2010). In (Spinello and Arras, 2011), 3 sensors are used to reconstruct a larger field of view. This kind of sensor is, however, sensitive to sun light and is therefore limited to indoor mapping.

\subsection{Spherical acquisition system}

To acquire augmented visual spheres, for large-scale environment mapping, a first multi-camera system has been initially proposed in (Meilland et al., 2011a). This system will not be detailed here but the interested reader can refer to that article. This sensor, shown in Figure 6(a) was composed of six wide angle stereo cameras, placed in a ring configuration so that dense stereo matching can be performed between each camera pair. This compact configuration allowed to build spherical photometric panoramas with a depth information associated to each pixel of the sphere. One of the major drawback of this system is that the angles between the optical axes are clearly diverging $\left(60^{\circ}\right)$, and wide-baseline stereo matching is necessary to handle the large differences in image resolution. Several dense matching algorithms have been tested, from standard block matching to more advanced approaches (Kolmogorov and Zabih, 2001; Ogale and Aloimonos, 2005; Hirschmuller, 2008; Tola et al., 2010; Geiger et al., 2010). (Hirschmuller, 2008) and (Geiger et al., 2010) performed fairly, but the large differences in resolution leads to several mismatches which results in sparse depth-maps. Since the depth information is necessary to correctly warp and blend the intensities onto the final sphere, the resulting photometric spheres were not fully dense.

To overcome the limitations of the first sensor, a second configuration has been studied. This sensor system, similar to the MARS sensor (Earthmine, 2009), is composed of three stereo pairs back-to-back in a vertical 


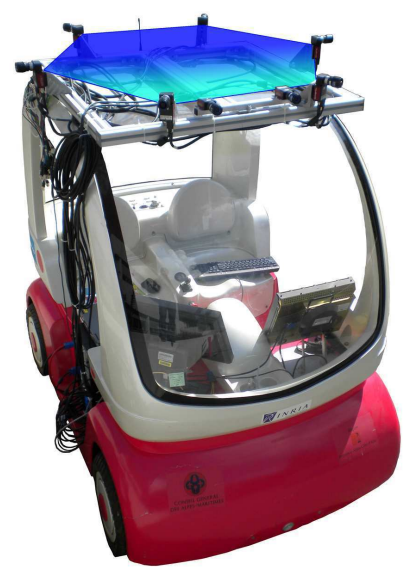

(a) Spherical sensor in ring configuration

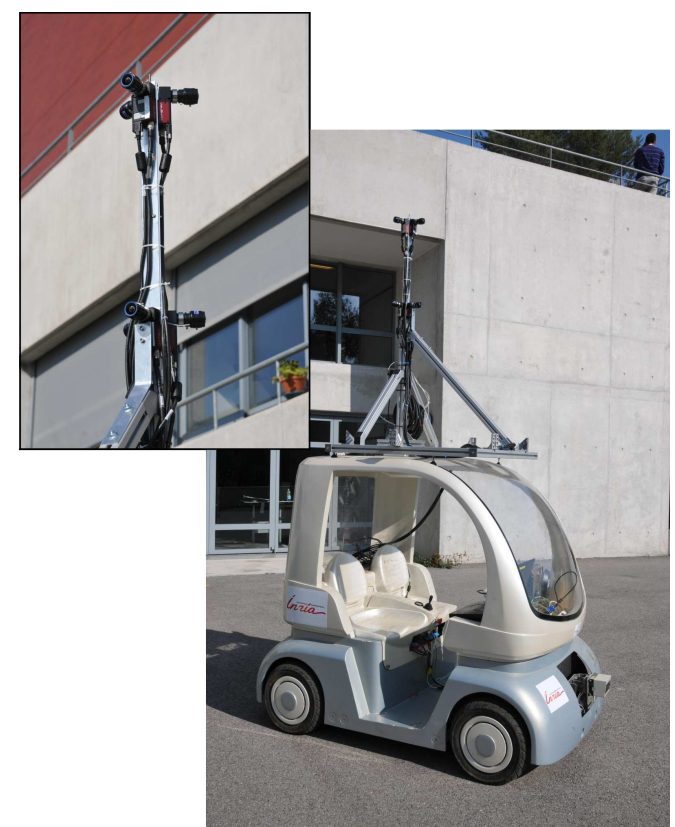

(b) Spherical sensor in vertical configuration

Figure 6: Spherical RGB-D sensors

configuration as it can be seen in Figure 6(b). Each triplet of cameras is considered to have a unique centre of projection, allowing to use standard stitching algorithms to build the photometric spheres. This configuration is similar to those using omnidirectional stereo cameras such as (Lui and Jarvis, 2010), except that using a multi-camera system allows to reconstruct high resolution spheres which are necessary for outdoor mapping. The procedure to build an augmented sphere with this system can be summarized as:

1. Calibration of the extrinsic and intrinsic parameters of each camera with a checker-board, via bundle adjustment: this calibration step is only performed once since the system is rigid.

2. For each triplet of cameras, perform image stitching with Laplacian blending (Burt and Adelson, 1983) which results in 2 spherical panoramas with a vertical baseline (top and bottom, see Figure 7).

3. Rectification of the spherical panoramas to ensure the epipolar constraints on the spheres.

4. Dense matching between the spheres.

5. Triangulation between the spheres to recover depth.

In this configuration, dense matching is much more robust since the sensor is composed of three classic fronto-parallel stereo pairs back to back. Since the images can be directly warped onto the spheres by 


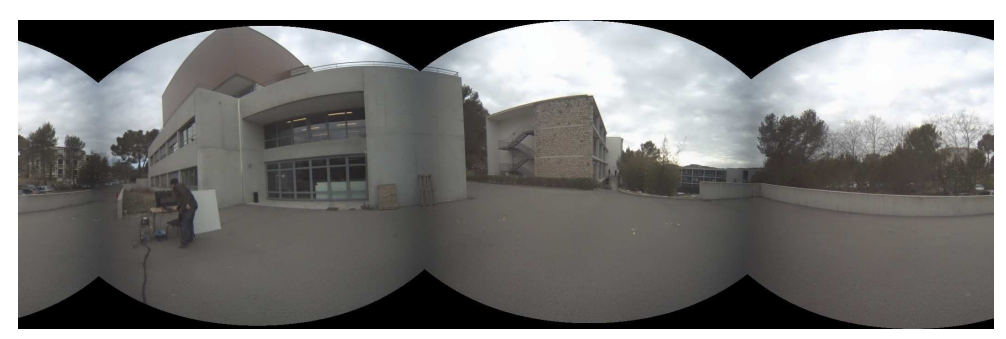

(a) Top spherical image

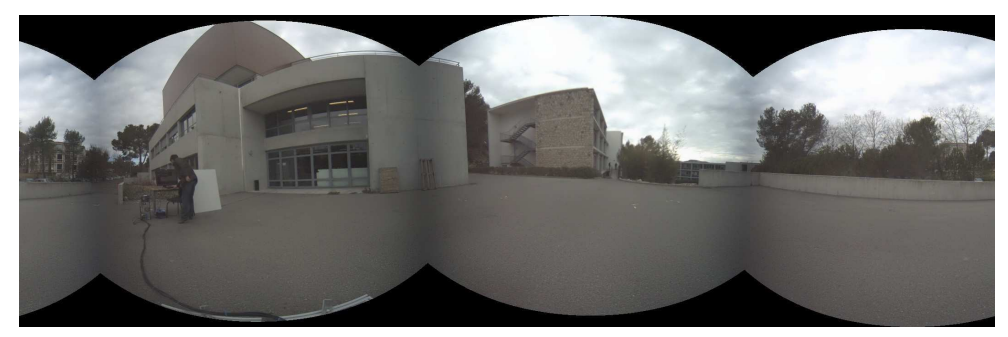

(b) Bottom spherical image

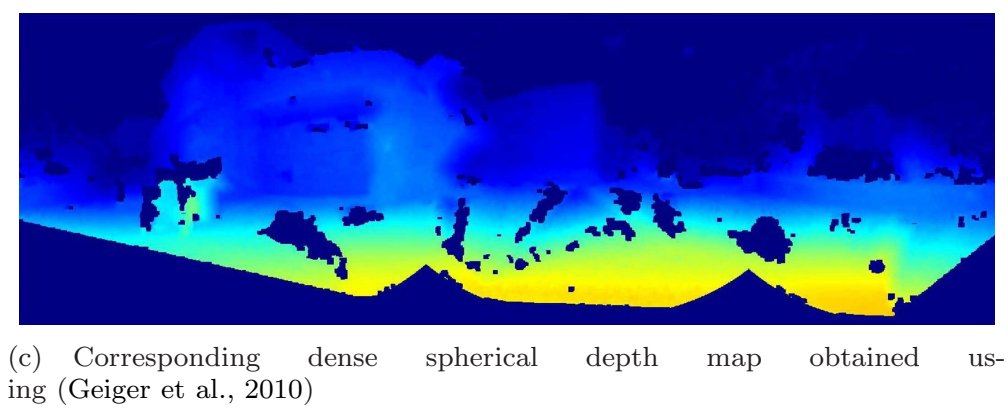

Figure 7: Example of an RGB-D panorama obtained using the new vertical system. 
stitching, the resulting photometric spheres are also dense. One potential issue is that due to the vertical alignment, vertical lines in the scene are collinear with the epipolar lines: this may create ambiguities during dense matching, especially in structured environments (in practice, this degenerated case was not observed in outdoor environments). Another issue with this system is the approximation of a unique centre of projection for each camera triplet which can lead to parallax artefacts when stitching the images. In practice those effects are only visible for very close objects $($ eg. $<1 m$ ), which is not an issue when mapping outdoor environments.

As it can be seen on Figure 7, this new system provides high quality photometric spheres and depth-maps, since dense matching can be performed directly between the spherical panoramas, leading to consistent results when using a semi-global approach such as (Hirschmuller, 2008) or (Geiger et al., 2010).

\section{Real-time localisation}

This section aims at presenting several techniques that have been used to improve the real-time performance of 6 dof pose estimation with respect to the previously described dense key-frame graph of augmented spherical images. First of all, it will be shown how the graph is used for real-time monocular pose estimation in an asymmetric manner (i.e. a single perspective image is compared to the closest augmented spherical image). Following that a multiple reference sphere localisation that provides a smooth localisation will be proposed along with a technique that improves robustness in dynamic environments. Finally an approach to first estimating only rotation before refining the full pose will be given that greatly improves convergence speed.

\subsection{Real-time monocular 6 dof localisation}

\subsubsection{Online sphere selection}

It is assumed that during online navigation, a current image $\mathbf{I}$, captured by a generic camera (e.g. monocular, stereo or omnidirectional) and an initial guess $\widehat{\mathbf{T}}_{\mathcal{G}}$ of the current camera position within the graph are available. In order to choose the closest sphere for tracking within the graph, it is necessary to define a metric. Contrary to non-spherical approaches, a sphere provides all viewing directions and therefore it is not 
necessary to consider the rotational distance (to ensure image overlap). The closest sphere is subsequently determined uniquely by translational distance between the initial estimate $\widehat{\mathbf{T}}_{\mathcal{G}}$ and the sphere's poses :

$$
\mathbf{T}_{\mathbf{S}}^{*}=\underset{\mathbf{i}}{\arg \min }\left(\left\|\mathbf{e}_{4}^{T}\left(\mathbf{T}_{\mathcal{G}}^{i}\right)^{-1} \widehat{\mathbf{T}}_{\mathcal{G}}\right\|\right) \quad \forall i \in \mathcal{G}
$$

where $\mathbf{e}_{4}=\left[\begin{array}{llll}0 & 0 & 0 & 1\end{array}\right]^{T}$ is a vector that extracts the translational part of a pose $\mathbf{T}$.

In particular this avoids choosing a reference sphere that has similar rotation but large translational difference which induces self occlusions of buildings and also differences in image resolution caused by distance (which affects direct registration methods).

\subsubsection{Efficient minimisation}

Since a sphere provides all local information necessary for 6 degrees of freedom localisation (geometric and photometric information), an accurate estimate of the pose is obtained by an efficient direct minimisation:

$$
\mathbf{e}(\mathbf{x})=\mathbf{I}\left(w\left(\widehat{\mathbf{T}} \mathbf{T}(\mathbf{x}) ; s\left(\mathbf{Z}_{S}^{*}, \mathbf{P}_{S}^{*}\right)\right)\right)-\mathbf{I}_{S}^{*}\left(s\left(\mathbf{Z}_{S}^{*}, \mathbf{P}_{S}^{*}\right)\right)
$$

where $\mathbf{x}$ is the unknown 6 degrees of freedom pose increment obtained as detailed in Section 2. The warping function $w(\cdot)$ transfers the current image intensities onto the reference sphere via both the perspective and spherical warping functions given in equations (26) and (29), using the depth information $\mathbf{Z}_{S}$ of the reference sphere. The function $s(\cdot)$, introduced in Section 3.6, selects only informative pixels, with respect to a saliency map $\mathbf{W}_{S}$ which is already pre-computed on the reference sphere and stored in the graph. This selection speeds up the tracking algorithm without neither degrading observability of 3D motion nor accuracy.

\subsubsection{Smooth localisation}

During this localisation phase, the live current image is permanently registered onto the closest reference sphere which is retained for localisation until a new sphere is selected according to criterion (17). As the current image get further from the reference image, the amount of occlusions and outliers increase, due to viewpoint and resolution changes, leading to localisation inaccuracies. This can create discontinuities in the trajectory when a new reference sphere is chosen (see Figure 9). Even if these discontinuities are small (e.g. few centimetres), they can impact the visual servoing used for autonomous driving. 


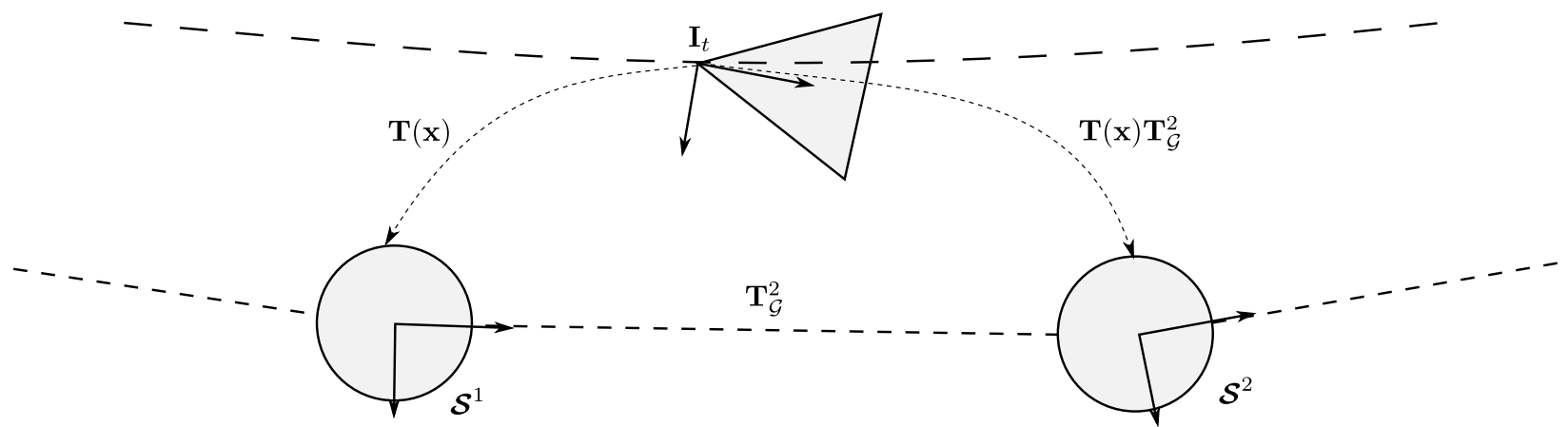

Figure 8: The image $\mathbf{I}_{t}$ is localised using simultaneously the nodes $\mathcal{S}^{1}$ and $\mathcal{S}^{2}$, which ensure a smooth localisation.

In order to obtain smoother trajectories and a more robust localisation, it is proposed here to use simultaneously two reference images in the minimisation, successively selected by the criteria (17). This usually corresponds to select the previous and next spheres in the graph as it is shown on figure 8 .

The localisation problem can be formulated as a simultaneous minimisation of two intensity errors:

$$
\mathbf{e}_{\mathbf{G}}(\mathbf{x})=\left[\begin{array}{c}
\mathbf{I}\left(w\left(\widehat{\mathbf{T}}_{\mathbf{S}} \mathbf{T}(\mathbf{x}) \mathbf{I} ; s\left(\mathbf{Z}_{S}^{1}, \mathbf{P}_{S}^{1}\right)\right)\right)-\mathbf{I}_{S}^{1}\left(s\left(\mathbf{Z}_{S}^{1}, \mathbf{P}_{S}^{1}\right)\right) \\
\mathbf{I}\left(w\left(\widehat{\mathbf{T}}_{\mathbf{S}} \mathbf{T}(\mathbf{x}) \mathbf{T}_{\mathcal{G}}^{2} ; s\left(\mathbf{Z}^{2}, \mathbf{P}^{2}\right)\right)\right)-\mathbf{I}_{S}^{2}\left(s\left(\mathbf{Z}_{S}^{2}, \mathbf{P}_{S}^{2}\right)\right)
\end{array}\right],
$$

where $\mathbf{T}_{\mathcal{G}}^{2}$ is the pose of the second reference sphere $\mathbf{S}^{2}$ with respect to the first reference sphere $\mathbf{S}^{1}$.

Using two reference images in the minimisation introduce more pixels in the cost function. In order to maintain high frequency tracking, the number of salient pixels used in the minimisation is simply shared between the spheres. Note that (8) can easily be expanded to more reference spheres, but it appears experimentally that two spheres are sufficient for smooth and robust localisation. Indeed using reference images that are too distant from the current view introduce additional occlusions which reduce the efficiency of the minimisation.

Figure 9 compares a single node localisation (red) with a multi-node localisation (blue). When using a single node, it can be seen that the trajectory is not continuous when the reference image is switched (around $X=-1, Z=5)$. When using simultaneously two nodes, the estimated trajectory remains smooth.

\subsection{Efficient estimation of local 3D rotations}

Typically, the apparent motion of pixels in an image is dominated by rotation motions. Indeed, for a pure rotation, the pixel's motion is independent of the scene geometry. For a vision based localisation algorithm, 

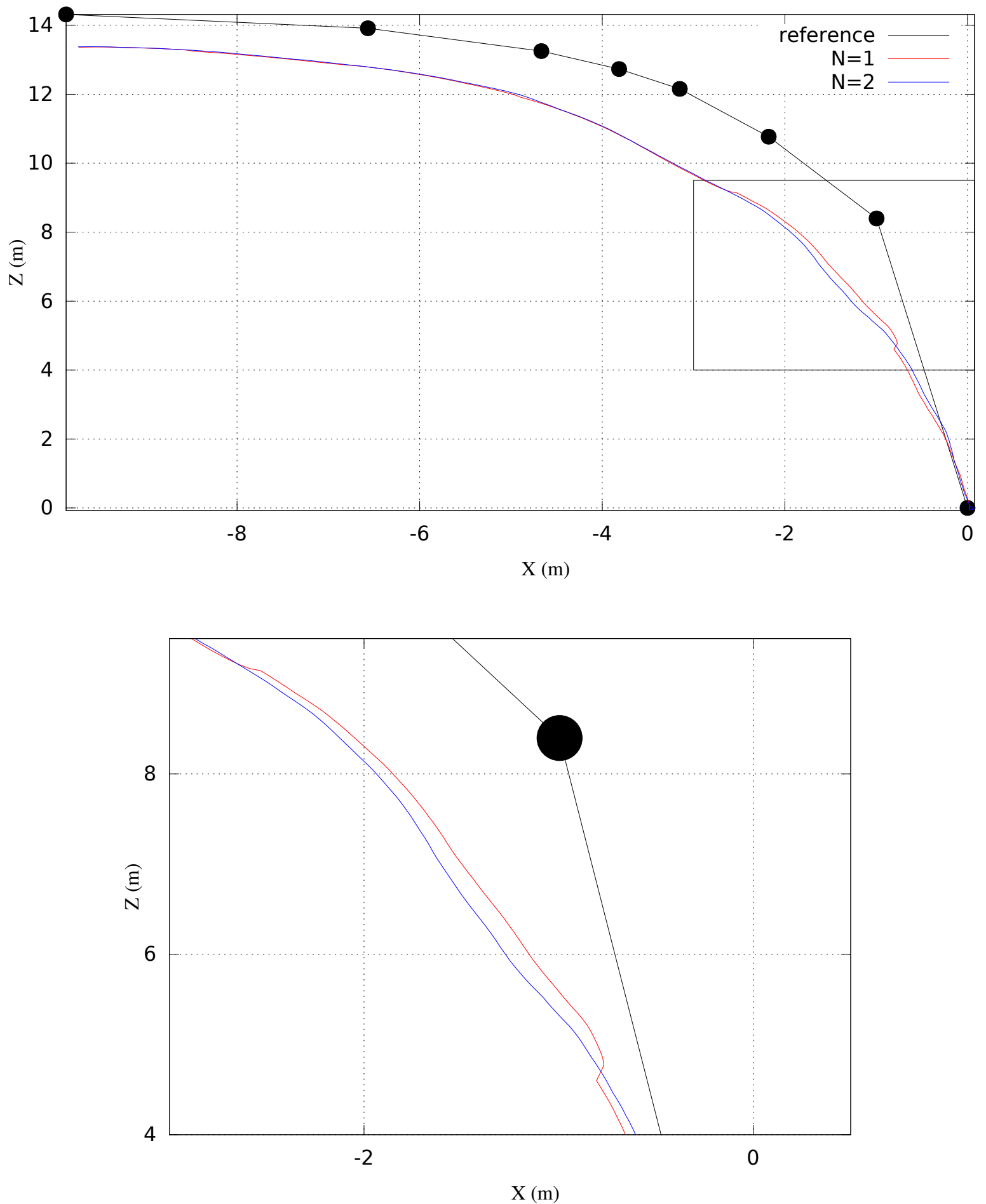

Figure 9: Multi-nodes localisation: The black disk represents the position of the reference spheres. In red the trajectory estimated using only the closest reference node. The trajectory contains discontinuities when switching reference. In blue the same trajectory, estimated using the 2 closest nodes. The obtained trajectory is continuous, which better represents the expected trajectory of the vehicle. 
large rotations between successive frames is often prone to failure. To ensure a better initialisation, a direct registration technique is employed to estimate the local inter-frame 3D rotation of the camera as in (Mei et al., 2010; Lovegrove and Davison, 2010; Newcombe et al., 2011b).

The smallest image in the multi-resolution pyramid at time $t$ is registered with the corresponding image at time $t-1$. Since those images are generated from successive Gaussian filters and sub-sampling, rotations are clearly dominant and translations can be neglected. The corresponding error is then obtained as follows:

$$
\mathbf{e}\left(\mathbf{x}_{\omega}\right)=\mathbf{I}_{t}\left(w\left(\mathbf{K} \widehat{\mathbf{R}} \mathbf{R}(\boldsymbol{\omega}) \mathbf{K}^{-1} ; \mathbf{P}\right)\right)-\mathbf{I}_{t-1}(\mathbf{P})
$$

where $\mathbf{K}$ are the intrinsics parameters of the camera, $\widehat{\mathbf{R}}$ is the initial rotation guess, $\boldsymbol{\omega} \in \mathbb{R}^{3}$ contains the unknown angular velocities, and $\mathbf{R}(\boldsymbol{\omega}) \in \mathbb{S O}(3)$ is obtained by the exponential matrix of $[\boldsymbol{\omega}]_{\times}$. This error can be efficiently minimised as described in Section 2.2, and allows to quickly recover large angular motions. Since a lower resolution image is used it is possible to perform dense minimisation (see computing times in table 1).

\subsection{Dynamic environment mapping}

In practice outdoor environments have very challenging dynamic effects that up until now have been handled as outliers, however, when both extreme illumination variations and dynamic moving objects are evolving within the scene the robust estimator breaks down. As such we have also created a further model for the illumination variations whereby a global parameter is estimated for changes such as the sun while local variations are still handled as outliers. To remain robust to dynamic changes, a temporal visual odometry error is added to the model-based error introduced in Section 5.1.2. This consists in simultaneously registering the current image $\mathbf{I}_{t}$ with the model $\mathbf{I}^{*}$ and with the last registered image $\mathbf{I}_{t-1}^{w}$. In this case the model-based error $\left(\mathbf{e}_{M B}\right)$ criteria ensures that the pose estimation does not drift but is not able to handle illumination change while the visual odometry error $\left(\mathbf{e}_{V O}\right)$ is invariant to the large illumination change (variation interframe at $45 \mathrm{~Hz}$ is considered negligible).

Figure 10 shows an example of hybrid minimisation, after registration of the current image for the experimental stereo dataset presented in Section 6.3.4. The scene contains both global illumination changes and local illumination changes, as well as dynamic occlusions (e.g. moving cars, bicycle, pedestrians). After registration, the model-based residual error $\mathbf{e}_{M B}$ is large, due to the saturation of the building façade on the left which is under-exposed in the reference image $\mathbf{I}^{*}$ and saturated in the current image $\mathbf{I}_{t}$ at time $t$. Large 
errors are also generated by moving vehicles, and by non-Lambertian reflections on the leafs of the trees. The associated M-estimator weights $\mathbf{D}_{M B}$ are rejecting most of the information. On the other hand, the residual visual odometry error $\mathbf{e}_{V O}$, which is defined between the last warped image $\mathbf{I}_{t-1}^{w}$ at time $t-1$ and the current image $\mathbf{I}_{t}$ is relatively low. Only moving objects are rejected by the M-estimator weights, which ensures a robust and fast convergence of the minimisation. More details can be found in (Meilland et al., 2011b).

\subsection{Initialisation and tracking failure}

To handle initialisation and tracking failure, a simple technique is employed. The current camera pose with respect to the reference sphere is supposed to be close to the identity. Dense registration is then performed with all the spheres of the graph, using the smallest image size in the pyramid. After alignment, the normalized cross correlation (NCC) is computed for each sphere of the graph, and the best score is used to select the reference sphere. Even if this initialisation step does not fit well with large-scale databases, it needs less than $30 \mathrm{~ms}$ per reference sphere, which is 9 seconds for a 300 spheres graph. A dedicated technique such as (Cummins and Newman, 2008; Chapoulie et al., 2011) will be investigated in future work.

\section{$6 \quad$ Field experiments}

In this section, several large scale field experiments involving each step of the proposed framework are presented. Firstly dense spherical environment mapping experiments are presented. Following this, realtime localisation and autonomous navigation experiments are detailed in the context of a national urban autonomous navigation challenge. Due to the overall complexity of the system and the breakup into a map learning phase and an online real-time navigation phase, it is suggested that the reader view the video associated with this paper before proceeding. The video illustrating these experiments can be found with the paper submission or alternatively at the following address http://youtu.be/wozzYVDQg2g.

\subsection{Implementation}

A real-time implementation of the online localisation algorithm has been made in $\mathrm{C}++$. Most parts of the code are SSE optimised (Steaming SIMD extensions), in order to take advantages of native vector instructions of modern processors. The iterative minimisation is performed in a multi-threaded environment. It involves computing a Gaussian pyramid of the current image, warping the current image, computing robust 


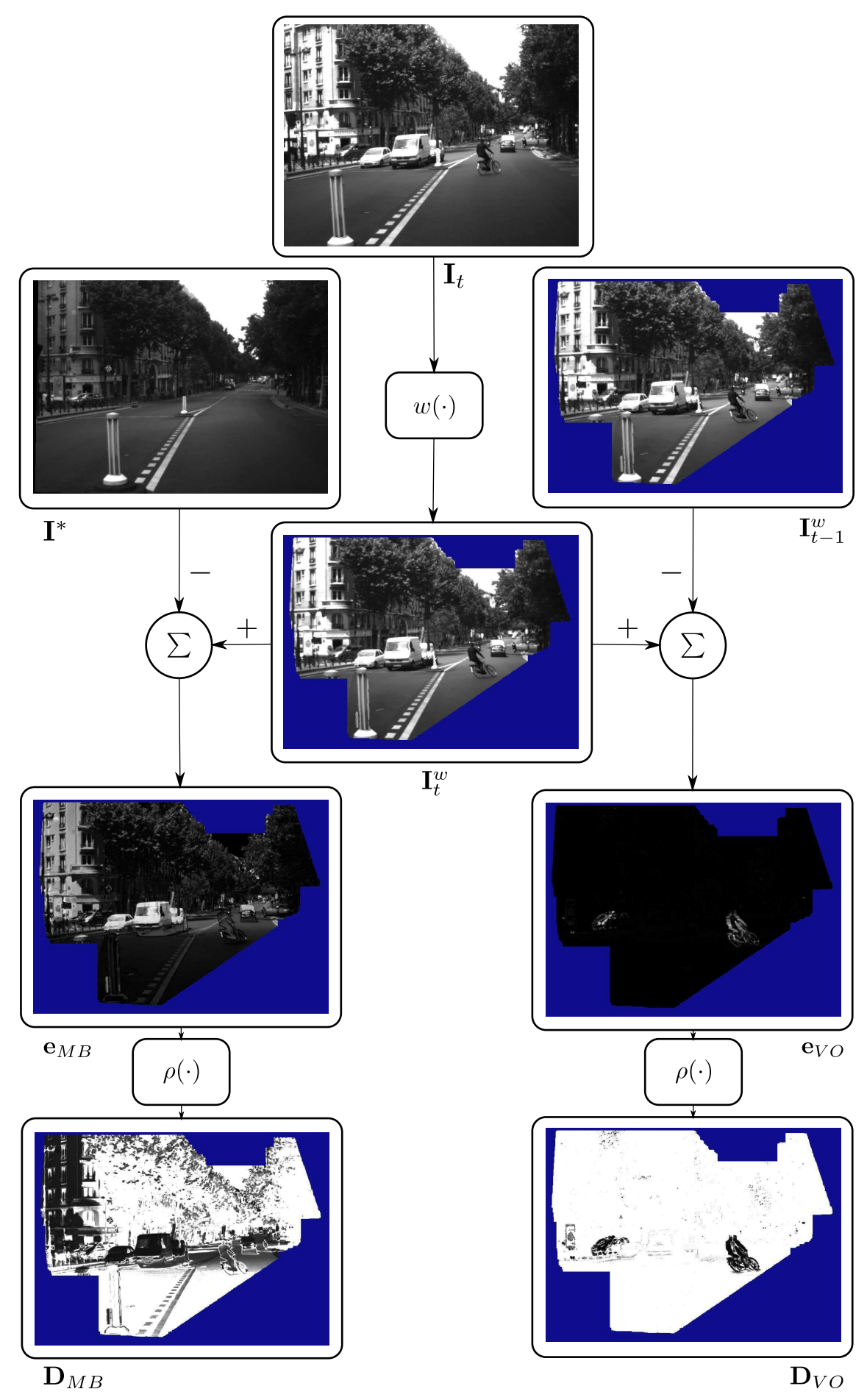

Figure 10: Hybrid visual tracking. In blue, depth information is not available in the reference image. On the left hand side, model-based registration (MB), the current image is registered wrt. the model, which contains large intensity errors $\left(\mathbf{e}_{M B}\right)$. The associated M-estimator weights $\mathbf{D}_{M B}$ are rejecting most of the information. On the right and side, visual odometry registration (VO), a temporally close error is minimised $\left(\mathbf{e}_{V O}\right)$. The M-estimator weights $\mathbf{D}_{V O}$, are only rejecting moving objects. 
statistics (median, MAD) using histograms, and computing the weighted Gauss-Newton Hessian and gradient approximations (see Section 2.2). The pose increment is then obtained using Cholesky factorisation of the $6 \times 6$ matrix, and the minimisation is iterated until convergence.

Using a small fraction of salient pixels (i.e. $60 \mathrm{k}$ pixels ), the localisation algorithm runs flawlessly at $45 \mathrm{~Hz}$ with an input image of dimensions $800 \times 600$ pixels. Table 1 shows the timings for each step of the algorithm obtained on a standard Core i7 laptop.

\begin{tabular}{|l|l|}
\hline Step & time $(\mathrm{ms})$ \\
\hline Reference images selection & $<0.01 \mathrm{~ms}$ \\
Gaussian pyramid & $0.70 \mathrm{~ms}$ \\
Inter-frame rotation estimation & $0.48 \mathrm{~ms} /$ iteration \\
6 dof localisation & $1.13 \mathrm{~ms} /$ iteration \\
\hline
\end{tabular}

Table 1: Computing time per step of the real-time localisation algorithm for $60 k$ pixels.

Since the localisation algorithm is based on an iterative minimisation, the number of iterations can be directly adjusted with the desired camera frame-rate. As it was highlighted in (Handa et al., 2012), a high camera frame-rate reduces the inter-frame camera motion, allowing to perform less iterations until convergence. In our experiments the maximum number of iterations for the inter-frame rotation estimation was set to 10 . The 6 dof localisation is performed using a 3 levels image pyramid with respectively $3,5,8$ iterations for each level of the pyramid (from coarse to fine).

\subsection{Storage cost and memory management}

As detailed in Section 3, each sphere contains a photometric image $\mathbf{I}_{S} \in \mathbb{R}^{+n \times m}$ which is directly converted into luminance and stored in a 16 bits grey level image, a set of pixel coordinates $\mathbf{P}_{S} \in \mathbb{R}^{2 \times n \times m}$ which is the same for all spheres, a 32 bits floating point depth map $\mathbf{Z}_{S} \in \mathbb{R}^{+n \times m}$ and a saliency map $\mathbf{W}_{S} \in \mathbb{N}^{n m}$ which contains a list of 32 bits unsigned integer indices. For each sphere a 3 level multi-resolution pyramid is also pre-computed. With a $2048 \times 1024$ base resolution, the cost of one sphere is 26.25 MB (without compression). For the large scale graph (310 spheres) reconstructed in Section 6.3.1, this represents 7.72 GB of memory. A better memory compression could be achieved using a lossless image compression algorithm. During online localisation, only a subset of the entire graph of spheres is loaded in RAM. Whilst the camera moves in the environment, a parallel CPU thread is used to stream out the farthest spheres and to stream in new spheres with respect to the current camera pose. This allows to navigate seamlessly in very large maps. 


\subsection{Localisation and mapping results}

\subsubsection{Dense mapping - INRIA Sophia Antipolis}

A sequence of $7364 \times 6$ images was acquired at $45 \mathrm{~Hz}$ on the site of INRIA Sophia-Antipolis with an electric Cycab vehicle and the on-board spherical system described in Section 4. The path travelled by the vehicle was approximately $1.5 \mathrm{~km}$ in length and is representative of a wide variety of outdoor environments including wide open spaces, buildings, canyons, parked vehicles, vegetation, trees, tight turns and varying altitudes. The aim of this being to test extensively the robustness and accuracy of the 6 degrees of freedom estimates of the vehicle pose. The learning phase which involved creating the augmented spherical panoramas and positioning them within a graph was calculated off-line at about $1 \mathrm{~Hz}$ due to the large amount of data to be handled.

The selection of specific key-frames and the detection of loop closures allowed to reduce the 7,364 initial images to 310 reference spheres with a compression rate of $96 \%$. Figure 11 shows the trajectory which was obtained after detecting loop closures and correcting the graph. Some of the key-frame images are also shown.

\subsubsection{Photo-realistic virtual navigation}

The final spherical representation can be used to synthesise photo-realistic virtual images, using a technique similar to those developed in image-based rendering ((Gortler et al., 1996; Levoy and Hanrahan, 1996; Debevec et al., 1996)). To demonstrate this, a real-time application was made with the OpenGL graphics rendering library which allows to navigate virtually within the key-frame graph by generating and rendering realistic novel views in real-time. This basic way of interacting with the proposed world representation illustrates the richness and quality of the model and also suggests possible alternative applications. Given a photo-realistic real-time visualisation system, the next sections will show how this type of model can be used to perform real-time pose estimation and autonomous navigation of a vehicle.

In order to generate images, a virtual camera is controlled manually by the user (keyboard and mouse) to define the 6 dof pose within the graph. The closest RGB-D sphere to the camera is then used to generate the view of the virtual camera using novel view synthesis. Figure 12 shows a virtual view that has been synthesised from a key-frame sphere. It can be noted that each sphere is valid for a local domain (in practice around $5 \mathrm{~m}^{3}$ ) until errors in the depth map begin to create visual distortion. It is therefore possible to generate an infinity of virtual images locally around the acquired nodes of the graph without having ever observed them before. 


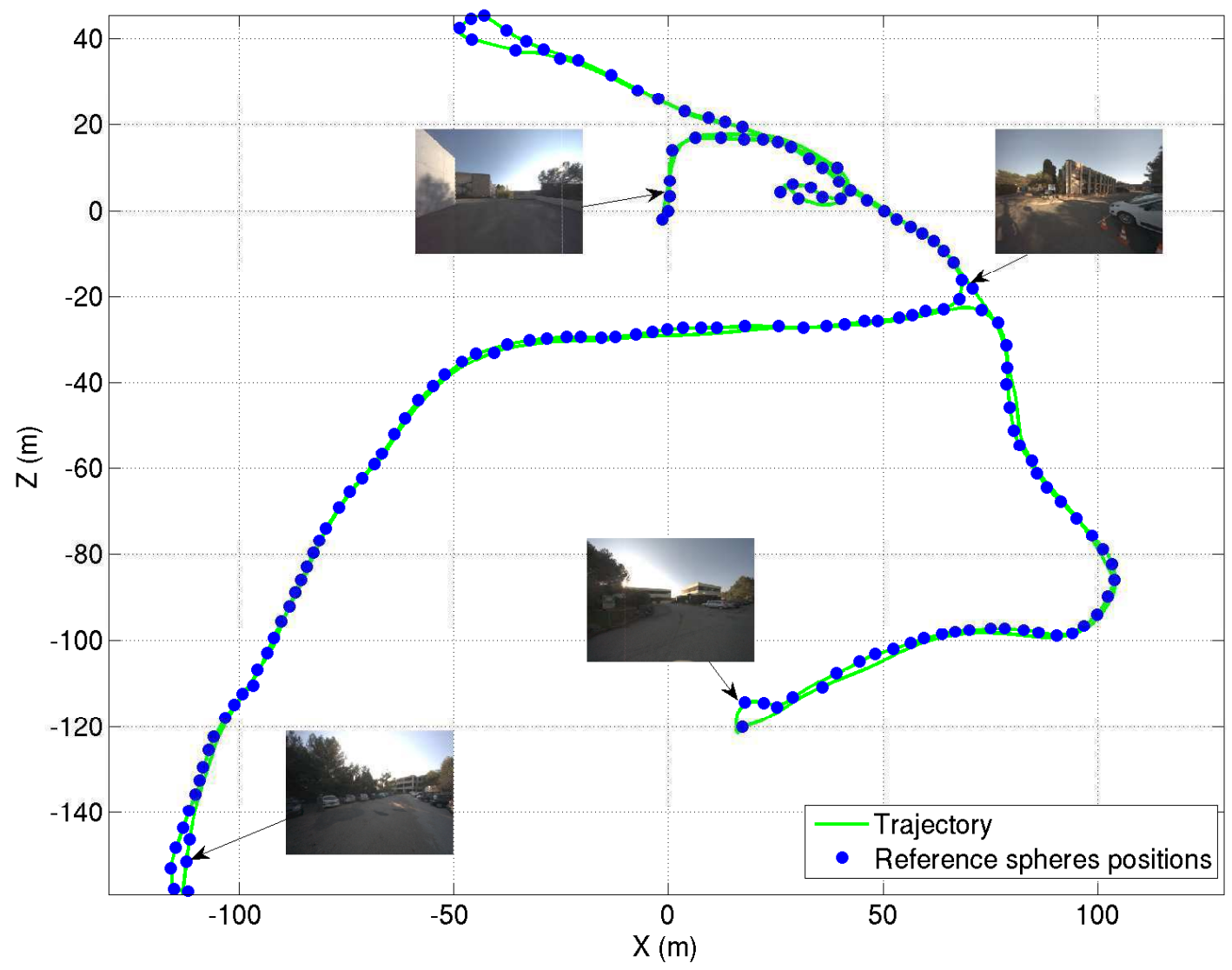

Figure 11: A spherical key-frame graph covering $1.5 \mathrm{kms}$.

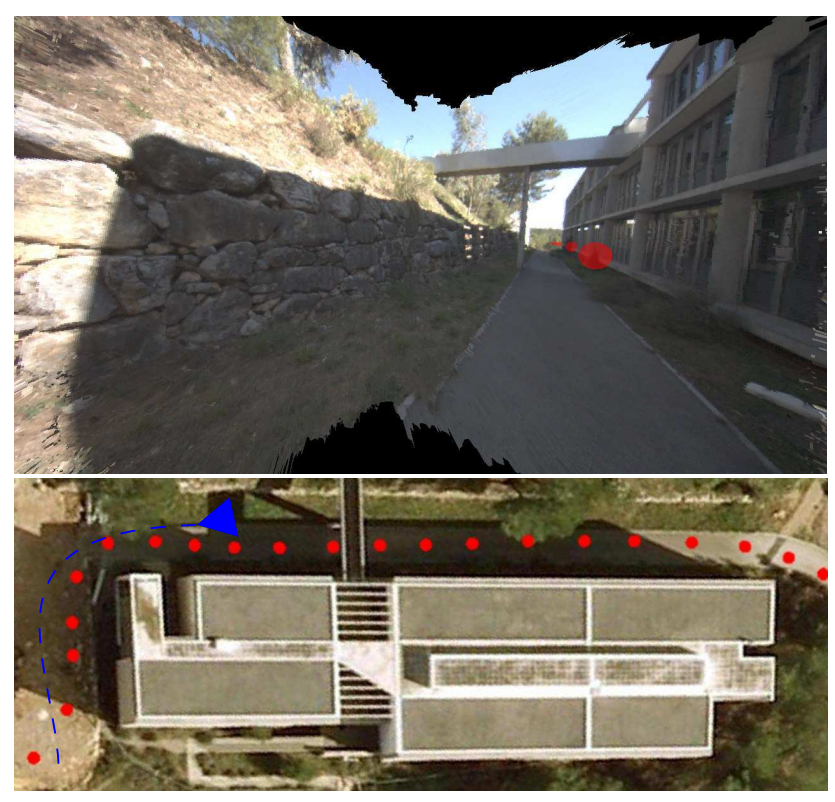

Figure 12: Top: Virtual image generated from an augmented spherical image. Bottom: An aerial view illustrating the global graph of key-frames. 


\subsubsection{Asymmetric real-time localisation}

One of the main advantages of a spherical key-frame graph is that any type of online sensor can be used to estimate the relative pose with respect to this environment model. In particular, whether the online sensor be monocular, omnidirectional, spherical RGB-D or other types of sensors, it is possible to define a warping function that allows to align each sensor data type with a spherical RGB-D graph. Here the term "asymmetric" is used to highlight this advantage. In practice both monocular and stereo online camera configurations were tested in order to perform asymmetric 6 dof localisation in real time with respect to a previously acquired spherical key-frame graph. The monocular camera is advantageous since it is low cost and only requires warping a single image (in exchange for faster computation but less robustness). The stereo camera pair, on the other hand, requires an extra image but is more robust to occlusions. Several real-time experiments were performed to validate the performance (robustness, precision, computational efficiency) of this asymmetric online localisation algorithm.

The first experiment was conducted using a single key-frame graph in order to test the domain of validity of a single sphere. A monocular camera with a resolution of $800 \times 600$ pixels, was manually moved around the sphere and online tracking was performed at $45 \mathrm{~Hz}$. Figure 13 shows the trajectory of the camera with respect to the reference sphere. It can be seen that using a spherical image allows to localise the camera in all viewing directions. In this practical setting (with given resolution and camera and scene configuration), high quality localisation can still be performed up to 3.5 meters away.

The algorithm was then validated on a subset graph of 12 spheres extracted from the results presented in section 6.3.1. These images where acquired from the same monocular camera configuration whilst mounted on an electric Cycab vehicle navigating locally within the graph.

Figure 14 shows the trajectory that was obtained by the localisation algorithm. In the experiment the starting point of the vehicle is at coordinates $(X=-0.4, Z=-0.1)$ corresponding to image 14(a). The vehicle then advances in the positive $Z$ direction until it reaches the point $(X=-1.8, Z=20.5)$. The vehicle then reverses (green part) until it reaches the coordinates ( $X=3.3, Z=18.8$ ), at which point it then returns in the forward direction towards the starting point. It can be seen that the use of this representation allows to instantaneously capture all viewing directions which is particularly useful for locating a vehicle that may navigate in two or more directions along a road. What is more is that the depth component allows localisation of the vehicle from trajectories not previously seen therefore allowing vehicles to deviate from the learnt path. 


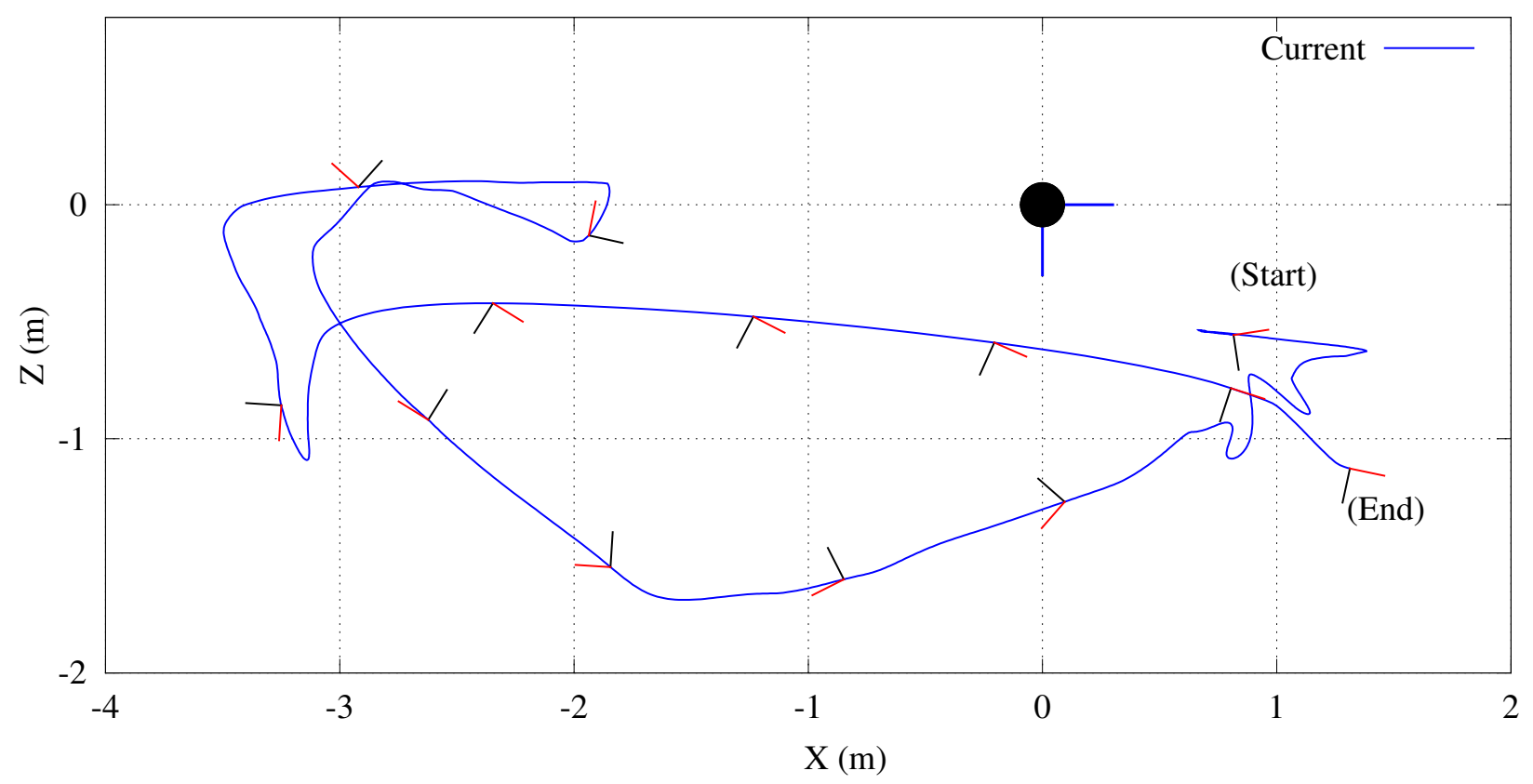

Figure 13: 6dof localisation around a spherical image. In blue: the trajectory of the estimated camera. Some of the orientations are displayed in red and black, where the optical axis is in red.

\subsubsection{Localisation in the $\mathrm{XII}^{t h}$ district of Paris}

The real-time 6 dof localisation method was also validated on large-scale stereo sequences captured in uncontrolled urban environments in the XII ${ }^{t h}$ district of Paris for the purpose of the French national ANR-CityVIP project. Whilst it can be argued that this type of environment is structured, the structure of this type of environment has not been explicitly exploited in the proposed method which also works in natural settings. Furthermore, the sequences were acquired on a vehicle operating normally amongst the flow of traffic (i.e. $50 \mathrm{~km} / \mathrm{h}$ ). The cameras used have a resolution images $800 \times 600$ pixels and acquisition is performed at $15 \mathrm{~Hz}$. At this frequency the displacement between successive images can be quite large and challenging, particularly when there are large rotations when the car takes a corner.

The topological key-frame graph was learnt via a first pass covering about $1 \mathrm{~km}$ in length. This resulted in a graph of 441 reference images. Since only stereo key-frames are used, a larger number of reference images is generated in the corners than the spherical model requires. This ensures sufficient overlap between the nodes of the graph ( $c f$. Figure 15).

Live localisation was performed from a second sequence of images that was recorded via a second pass which was significantly different from the first one due to the moving traffic, the different trajectory taken on the 


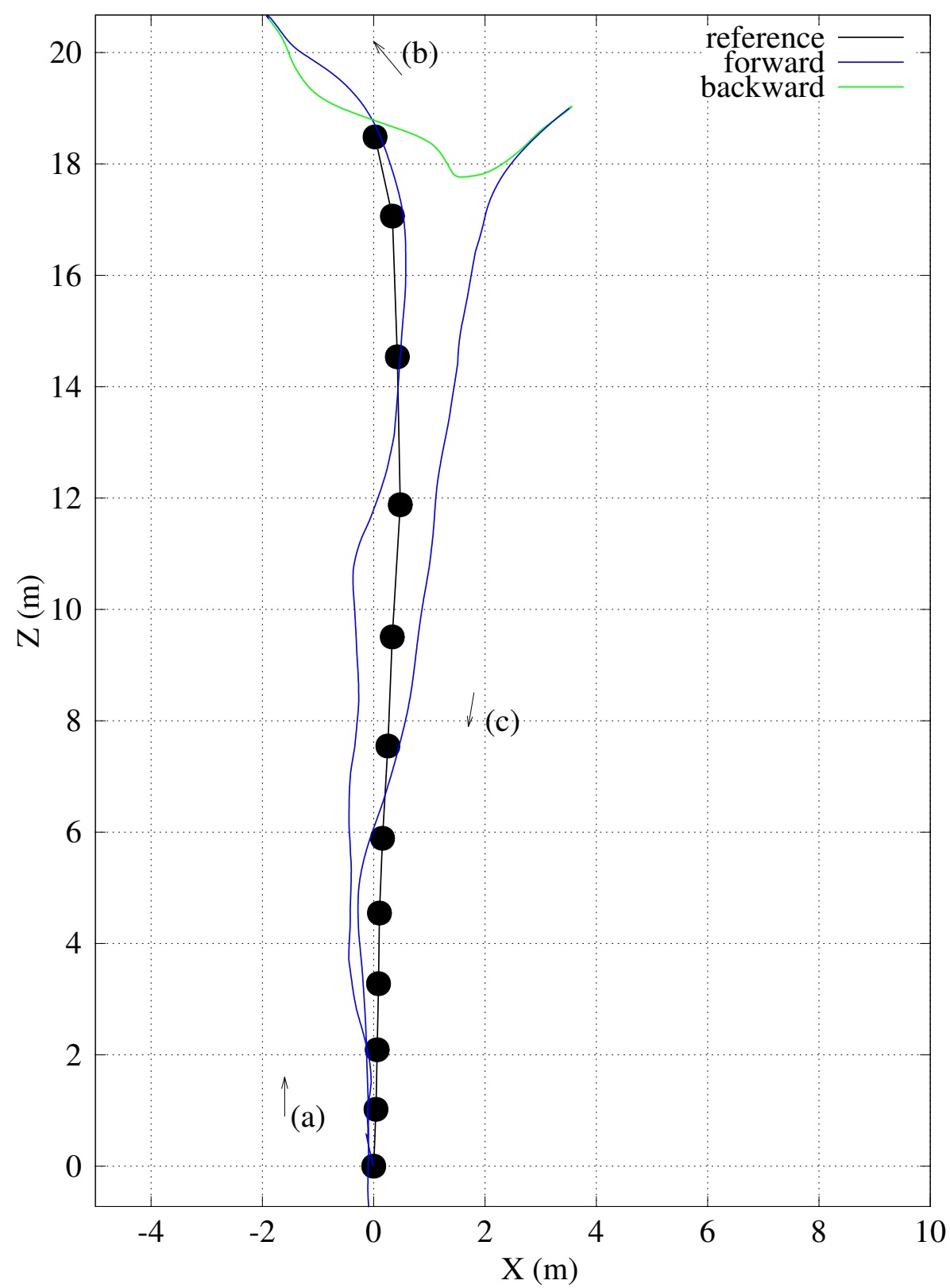

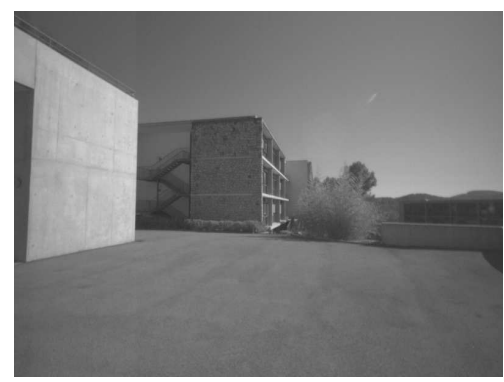

(a)

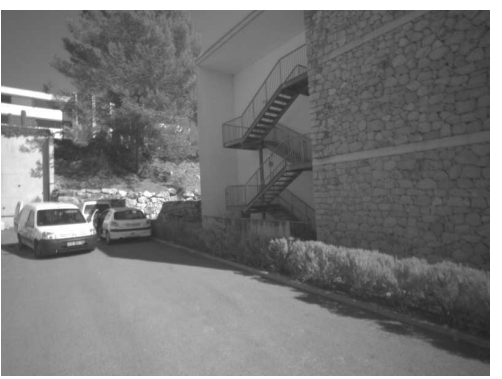

(b)

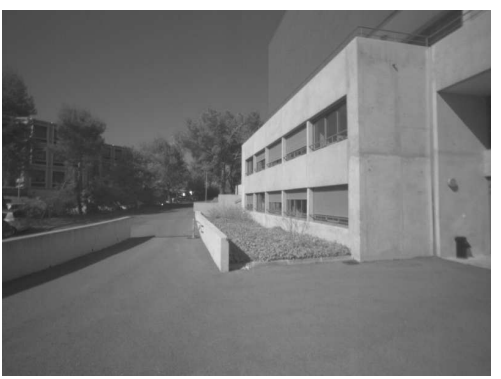

(c)

Figure 14: Localisation in a graph of RGB-D spheres. In blue: The vehicle is moving forward. In green: the vehicle is reversing. (a),(b) and (c), are images acquired during the live real-time tracking phase. 
road, along with different illumination conditions. Figure 15 shows the obtained trajectory and the position of the reference images. Although the vehicle is following the same road, the path is locally different from the learnt trajectory (the car overtakes, the turns are taken much more widely, etc). As it can be seen in sub-images (a),(b) and (c), the environment contains a lot of vegetation, illumination change, moving cars and pedestrians that are not in the database. Thanks to the M-estimator outlier rejection and the hybrid minimisation, the proposed localisation algorithm remains very robust to large changes in the scene. Furthermore the utilisation of inter-frame rotation estimation, defined in 5.2, allows to efficiently deal with large motion between the images (e.g. an inter-frame rotation of 5 degrees generates a motion of 50 pixels in the image).

\subsection{CityVIP autonomous driving challenge}

This section reports the autonomous navigation results obtained during the final challenge of the French ANR CityVIP project. This project was conducted by several leading French research teams from June 2008 to December 2011 and its aim was to develop autonomous transportation vehicles for urban environments. The goal of the challenge was to autonomously follow a reference trajectory over large scales in a completely uncontrolled environment. The entry presented here was based on a learnt environment map and trajectory acquired during a manual teaching phase. Whilst the navigation path was mapped using a key-frame approach, the environment remained highly unstructured and included traffic (cars, bicycles, trams), pedestrians etc. In a first part, the platform will be presented before introducing the control law that was used for trajectory following. In a second part, the preliminary trials and preparations are first presented before detailing the final results for the autonomous driving challenge.

\subsubsection{Platform}

The platform used for the experiments is an electric Cycab vehicle (see figure 16(b)) which can be controlled using longitudinal velocities and steering angle. A Lidar is placed in the front of the vehicle and is only used to detect imminent collisions. A single Firewire camera, placed on the top of the vehicle, is used for online localisation. All the computations and control commands are performed on a Core i7 standard laptop, embedding the pre-computed graph of augmented spheres. Additionally an IP camera is used to broadcast a video to a ground station. 


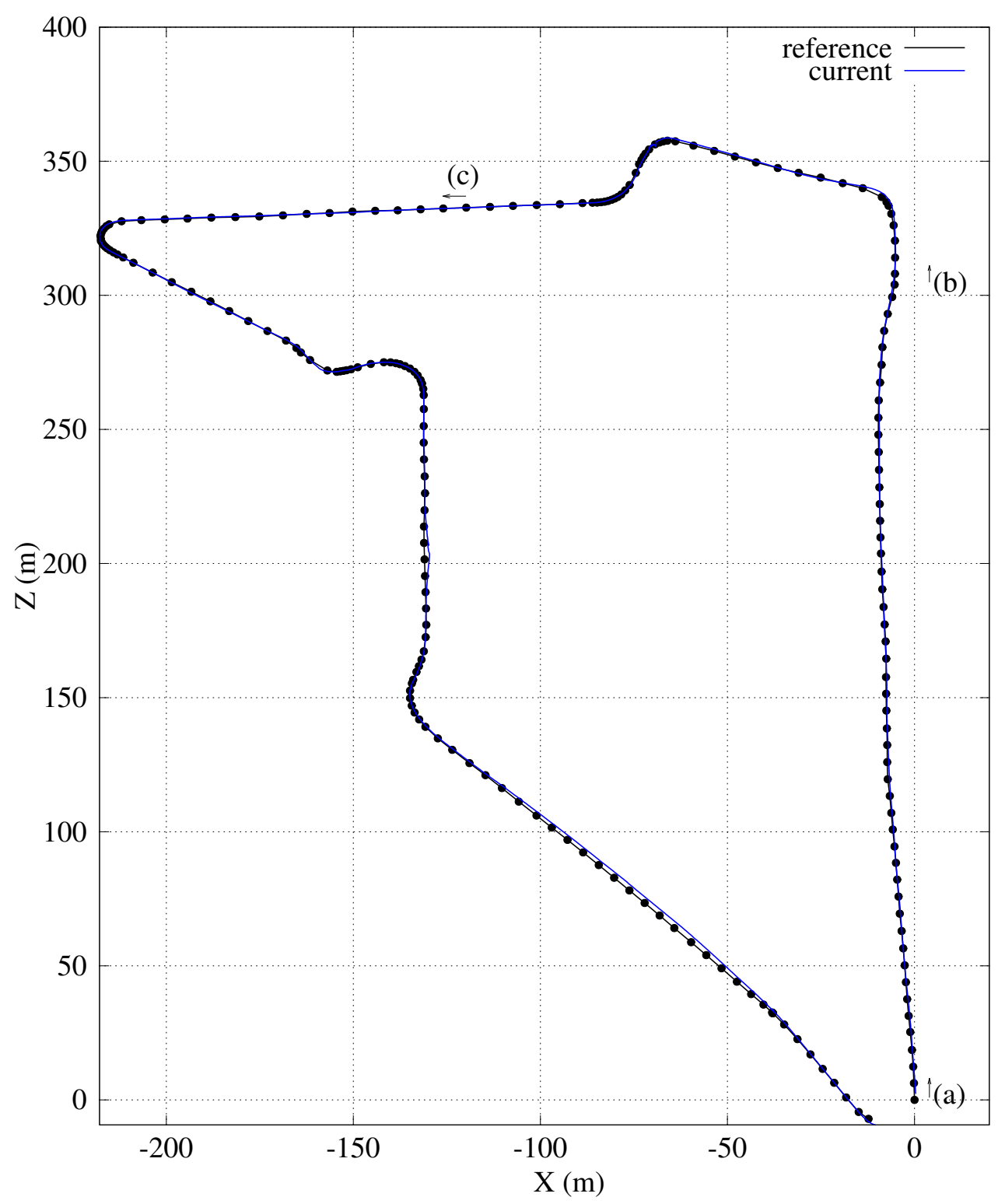

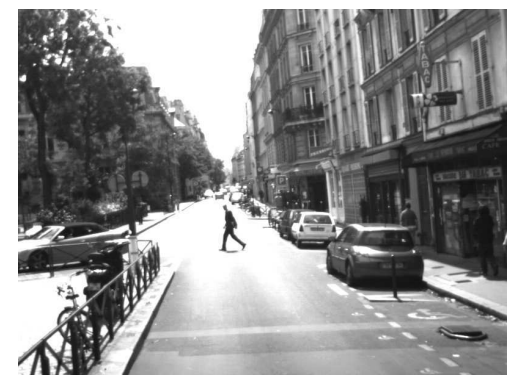

(a)

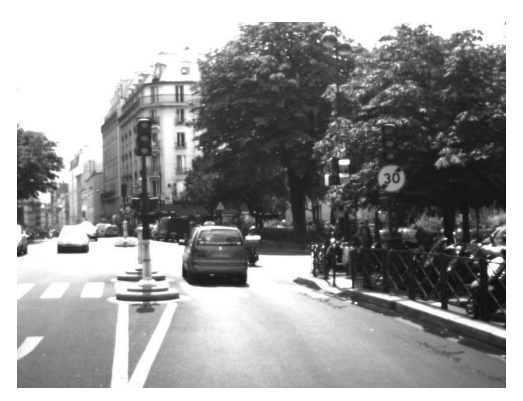

(b)

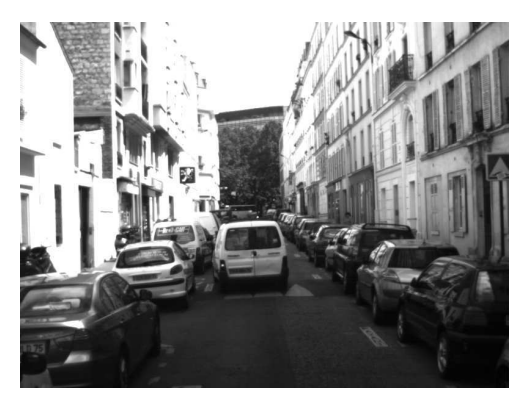

(c)

Figure 15: Localisation from a key-frame graph in the XII ${ }^{\text {th }}$ district of Paris. In blue: the trajectory which was estimated by the real-time localisation algorithm. In black, the position of the reference images (only one out of two reference images is shown). (a),(b) and (c), are images which were acquired during the on-line localisation phase. 


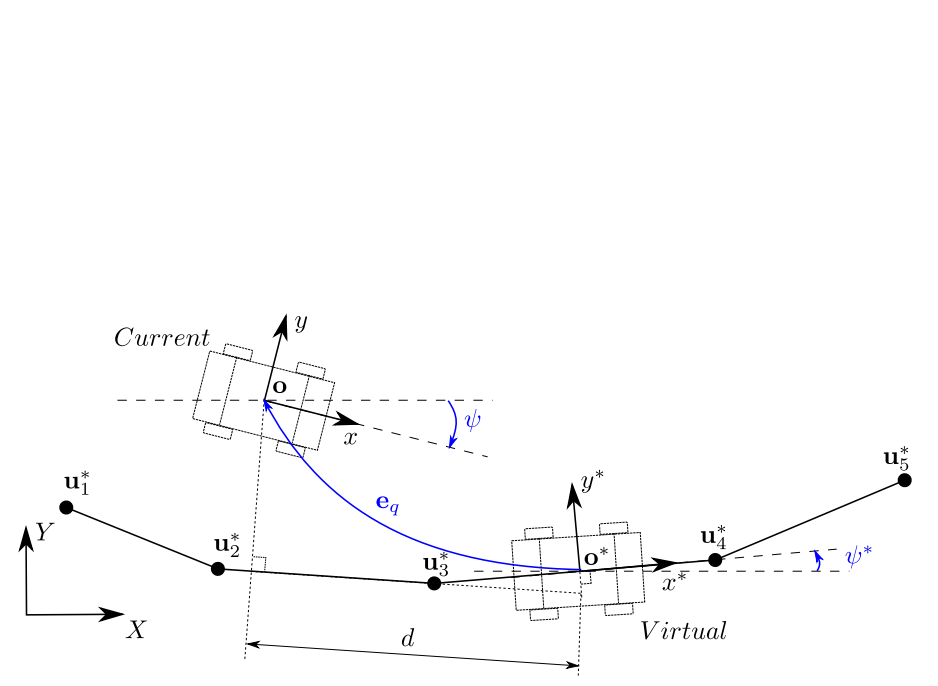

(a) Trajectory following

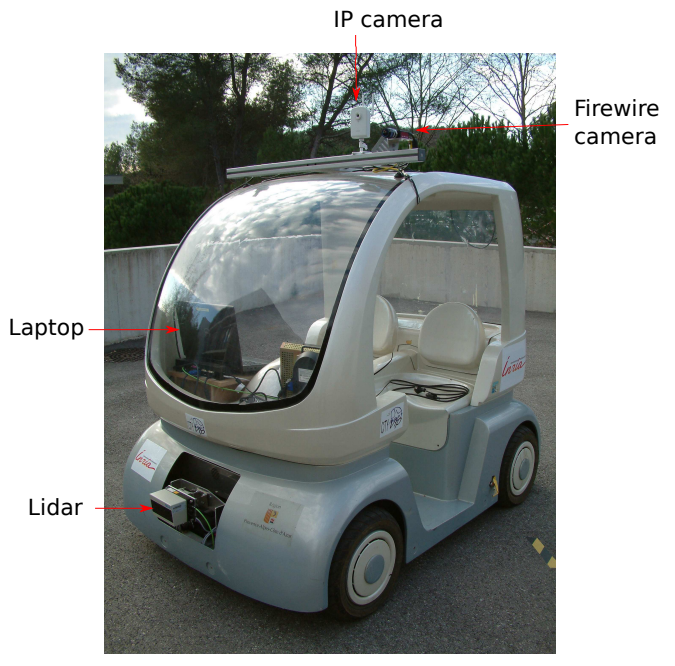

(b) Platform

Figure 16: (a) Regulated error for visual servoing. The current vehicle position is projected onto the closest reference trajectory's edge. A reference position is selected at a distance $d$, to generate longitudinal and angular errors $\left\{\mathbf{e}_{q}, e_{\psi}\right\}$. (b) Cycab vehicle with its sensors. The Firewire camera is placed on the top of the vehicle. The Lidar sensor is only used to detect obstacles.

\subsubsection{Control aspects}

For autonomous driving, the aim is to follow automatically a reference trajectory $\mathcal{U}$ generated locally around the learnt graph. The trajectory $\mathcal{U}=\left\{\mathbf{u}_{1}^{*}, \mathbf{u}_{2}^{*}, \ldots, \mathbf{u}_{n}^{*}\right\}$ contains $n$ input vectors such that:

$$
\mathbf{u}^{*}=\left\{x^{*}, y^{*}, \psi^{*}, U^{*}, \dot{\psi}^{*}\right\}
$$

where the point $\mathbf{o}^{*}=\left\{x^{*}, y^{*}\right\}$ is a desired position, $\psi^{*}$ is the yaw angle, $U^{*}$ is the longitudinal velocity and $\dot{\psi}^{*}$ is the desired angular velocity.

The control problem can be formulated as detailed in (Benhimane et al., 2005). In the proposed case, a virtual vehicle is followed and used to generate an error in translation and orientation that can be regulated using state feedback. Longitudinal velocity is controlled using a proportional feedback on the longitudinal error and steering angle depends on yaw and transversal errors. These errors are obtained by projecting the current vehicle position onto the closest reference trajectory's edge (see Figure 16(a)). The reference position is then selected by translating the projected position along the trajectory by a distance $d$. The translation error between the reference point and the current position is then defined by:

$$
\mathbf{e}_{q}=\left[\begin{array}{c}
e_{x} \\
e_{y}
\end{array}\right]=\mathbf{R}_{\psi^{*}}^{T}\left(\mathbf{o}-\mathbf{o}^{*}\right)=\mathbf{R}_{\psi^{*}}^{T}\left[\begin{array}{c}
x-x^{*} \\
y-y^{*}
\end{array}\right]
$$


where the rotation matrix of $\psi^{*}$ can be written by:

$$
\mathbf{R}_{\psi^{*}}\left[\begin{array}{cc}
\cos \left(\psi^{*}\right) & -\sin \left(\psi^{*}\right) \\
\sin \left(\psi^{*}\right) & \cos \left(\psi^{*}\right)
\end{array}\right] .
$$

The angular error is directly defined such as:

$$
e_{\psi}=\psi-\psi^{*}
$$

and the control law, derived from (Benhimane et al., 2005) is:

$$
\left\{\begin{array}{l}
U=U^{*}-k_{x}\left(\left|U^{*}\right|+\epsilon\right) e_{x} \\
\dot{\psi}=\dot{\psi}^{*}-k_{y}\left|U^{*}\right| e_{y}-k_{\psi}\left|U^{*}\right| \tan \left(e_{\psi}\right)
\end{array}\right.
$$

where the gains $k_{x}, k_{y}, k_{\psi}$ and $\epsilon$ are positive scalars.

\subsubsection{Challenge preparation and testing}

Preliminary autonomous navigation results were conducted at INRIA Sophia Antipolis in order to test the entire autonomous navigation pipeline. The following result reports autonomous driving on a small trajectory of 100 meters, which was manually generated around a pre-learnt key-frame graph. As it can be seen on Figure 17, the trajectory contains two 90 degrees turns. The reference vehicle longitudinal velocity was set to $1.4 \mathrm{~m} / \mathrm{s}$.

Figure 18 plots the longitudinal and transversal errors with respect to the time. It can be seen that the vehicle was able to follow the reference trajectory, keeping a longitudinal error close to zero, whilst the transversal error is less than $25 \mathrm{~cm}$. Note that the peaks in transversal errors correspond to the 90 degrees turns, which can be avoided with a better setting of the control gains of equation (25).

\subsubsection{Final CityVIP challenge}

The following section will present one of the many experiments conducted during the final CityVIP challenge that lasted one whole week at Place Jaude in the city centre of Clermont Ferrand, France. Over the course of one week, various trials were conducted in a large range of highly varying situations, allowing 


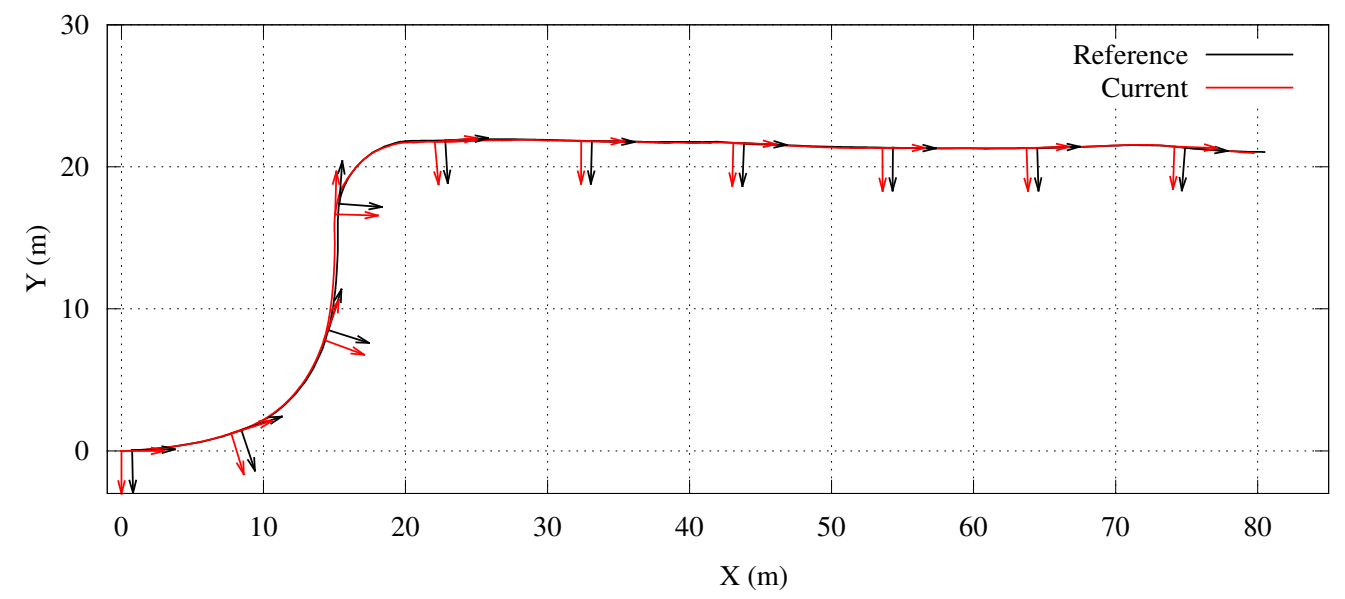

Figure 17: Trajectory autonomously followed at INRIA Sophia Antipolis. A subset of reference and current poses is plotted. The offset between the current and reference poses is related to the distance $d$ used to generate a longitudinal error. Corresponding longitudinal and transversal error can be found on Figure 18.

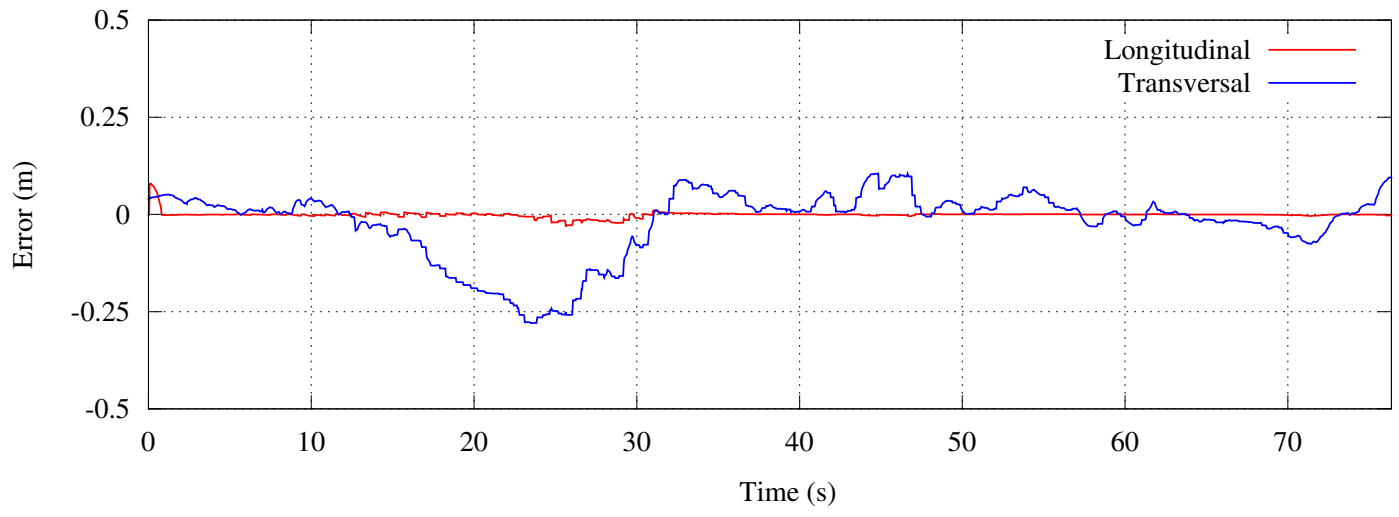

Figure 18: Longitudinal and transversal errors. The longitudinal error is centred around $d$. 
validation of the proposed approach. The intensive battery of tests included realistic, dynamic and largescale environments involving wide open spaces, narrow corridors, vegetation, trees and "hostile" moving objects such as pedestrians, bicycles and other vehicles.

A learning phase was performed along a 490 meters trajectory, by manually driving an electric Cycab vehicle equipped with the spherical acquisition system described in Section 4. In order to ensure an admissible path for the online navigation phase (i.e. without static obstacles), the trajectory obtained during the learning phase was used as input for the online navigation. Even so, it should be highlighted that the proposed localisation method is capable of deviating from the learnt path and can accurately estimate the camera pose within a local region around the graph, as it was demonstrated in Section 6.3.3. The reference longitudinal velocity was set to $1.2 \mathrm{~m} / \mathrm{s}$ for the whole sequence.

Figure 19 shows the desired trajectory in black, and the trajectory followed autonomously by the vehicle in red. The vehicle starts at position $(\mathrm{X}=0, \mathrm{Y}=0)$ and begins to move along $\mathrm{Y}$ axis. The experiment finishes at position $(\mathrm{X}=-62, \mathrm{Y}=61)$. The vehicle was able to follow autonomously the whole sequence, using only the monocular camera for localisation. As it can be seen on Figures (a),(b) and (c), the accuracy of the localisation method allows to navigate in narrowed corridors, whilst the M-estimator ensure robustness to occlusions like pedestrians. Images (d) and (f) show the vehicle navigating in much larger areas (open place). This kind of environment has shown some limitations of vision-based only navigation. Since geometric information is far from the camera (building façades), accurate estimations of translations are degraded (infinite points are invariant to translations). These effects can be seen on the red trajectory around the landmark (e). However, this lack of precision could be overcome using additional sensors, such as GPS and inertial measurements.

\subsubsection{Discussions on the experiments}

As mentioned in the previous section, visual navigation in large open-spaces is challenging since translations are not accurately estimated. This is particularly a problem when using wide angle cameras since a small translation of the sensor will not generate any changes in the images. On the other hand wide angle cameras provide more robustness. It could be interesting to combine a wide angle camera for robustness with a long focal camera for accuracy.

A second scenario has shown one limitation of the visual localisation approach. On figure 20(a), $10 \%$ of the best salient pixels of the image are shown in red. We can see that the strong gradients generated by 


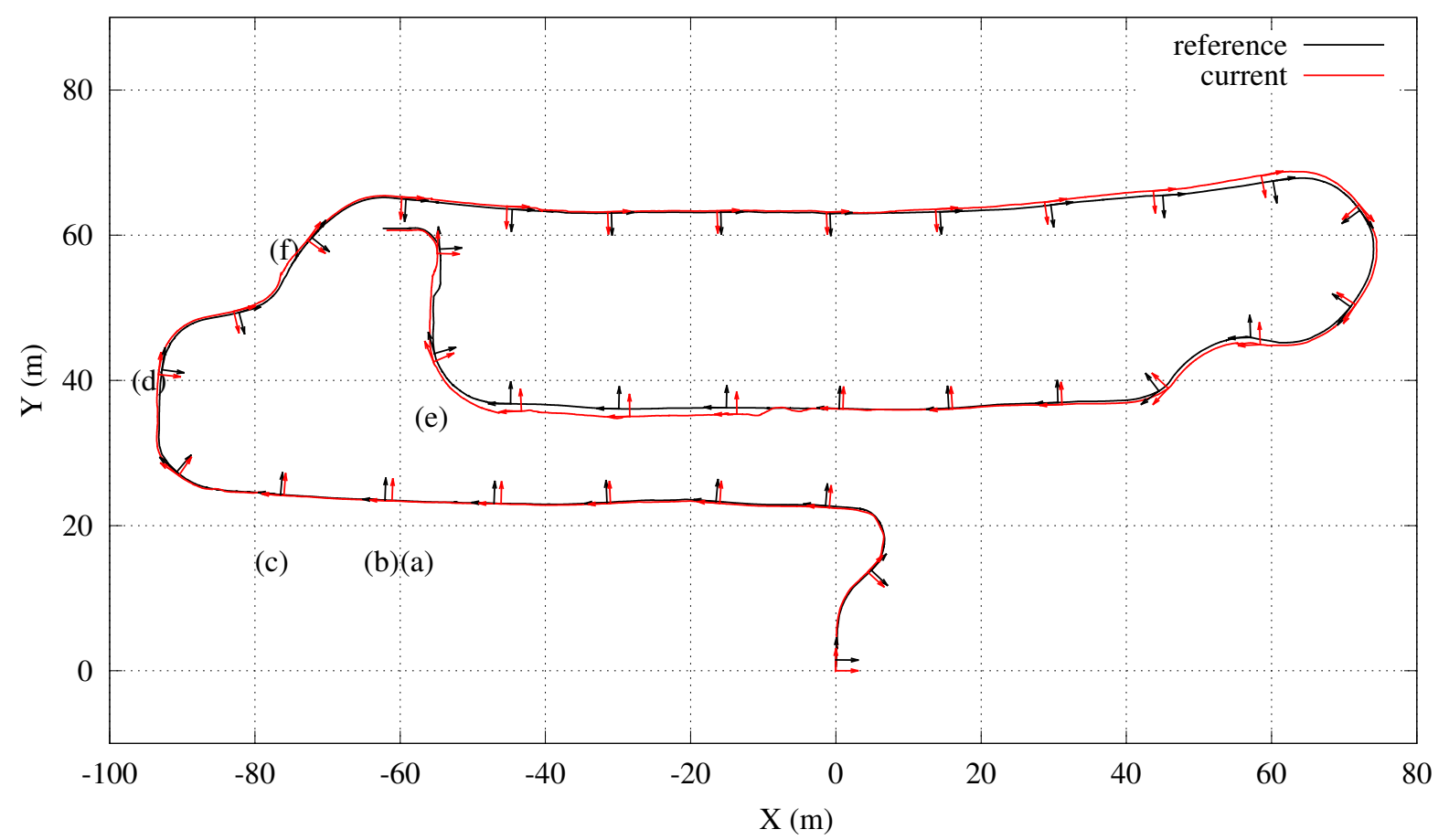

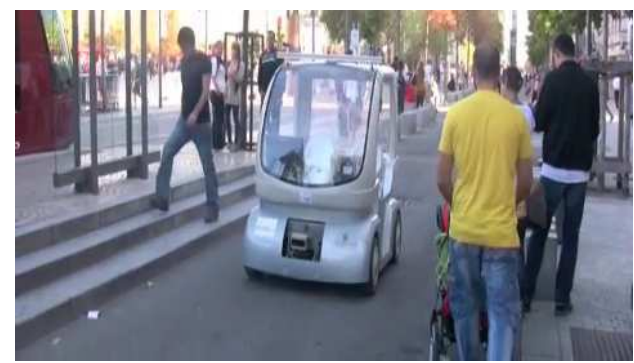

(a)

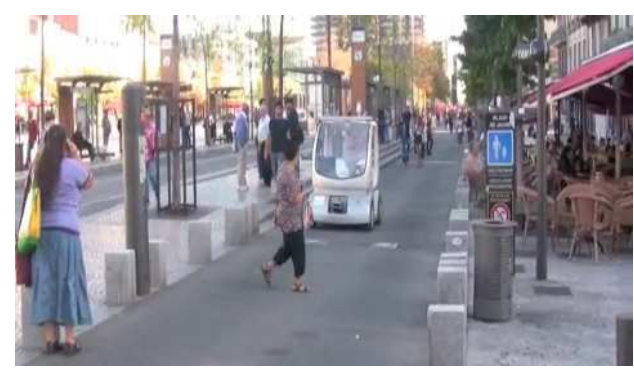

(c)

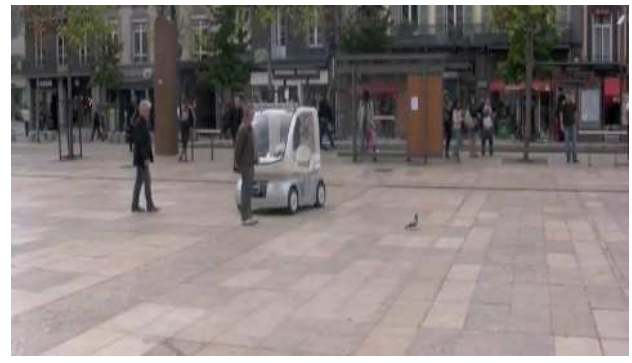

(e)

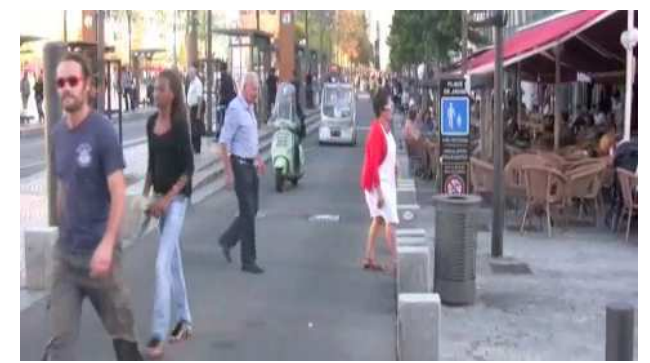

(b)

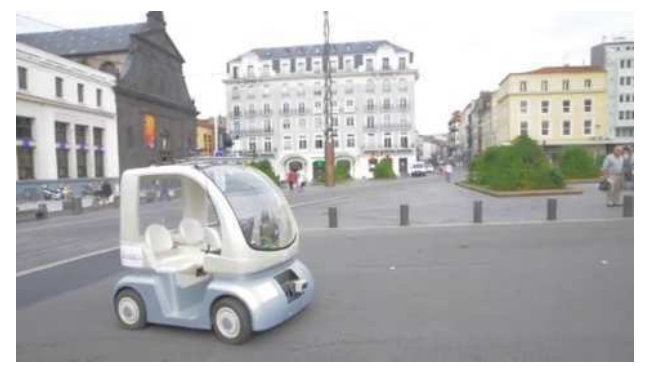

(d)

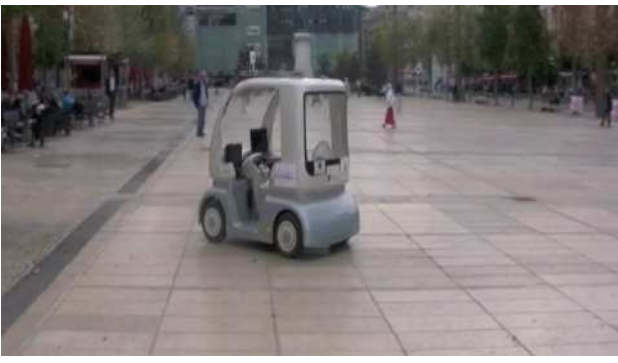

(f)

Figure 19: Autonomous navigation. Top: A 490 meters trajectory followed autonomously, the reference trajectory is shown in black. The current trajectory is shown in red and some virtual/current vehicle poses are plotted. (a),(b),(c),(d),(e),(f): images captured during the navigation. 


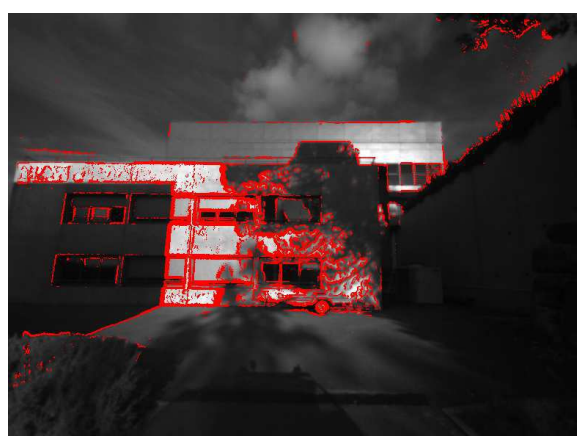

(a) $10 \%$ of the best salient pixels of an image

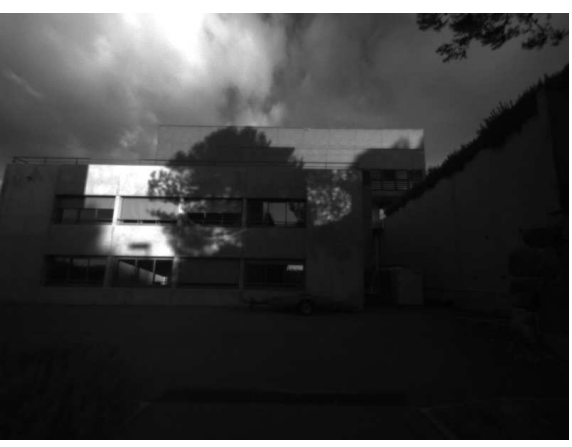

(b) Same scene observed one hour later.

Figure 20: Shadows example. Image (b) was acquired one hour after image (a). Strong shadows are selected as salient pixels.

the shadows are selected by the saliency selection algorithm. On figure 20(b), it can be seen that one hour later, the shadows are completely different. Even though the localisation approach is extremely robust, the minimisation can be perturbed by those false gradients. A simple solution could be to re-compute a saliency selection online wrt. to the outlier rejection. A second possibility could be to detect the shadows and use an invariant representation as in (Corke et al., 2013).

\section{Conclusions and future work}

The approach described in this paper proposes a complete mapping, localisation, guidance and control framework for autonomous navigation of robots in large unstructured environments. The mapping method allows to reconstruct dense visual maps of large scale 3D environments, using a novel spherical key-frame and topological graph representation combining photometric colour and depth information (RGB-D). It has been shown how to acquire this model using a learning approach and subsequently synthesising photometrically accurate views locally around the learnt graph. Reconstructed spheres acquired along a trajectory are used as input for a robust dense spherical tracking algorithm which estimates the spheres' positions.

During online navigation, an efficient direct registration technique is employed to accurately localise a monocular camera navigating within the graph. The robustness of the localisation method has been validated in challenging non-controlled sequences containing a lot of dynamic objects including traffic, pedestrians, lighting variation and other outliers. The proposed spherical environment map is asymmetric in that it can be used not only for the localisation of standard monocular cameras but also for stereo or omnidirectional systems. The proposed approach is, however, purely vision based in order to demonstrate the potential of 
such a low cost system, however, the system could easily be fused with other types of sensors such as IMU or GPS.

Future work will aim at improving the database construction, by geo-referencing the spherical graph in a GIS (Geographic Information System), which may contain higher level information such as free space. This geolocation will allow to use advanced path planning algorithms. To further improve the autonomous navigation solution, it should be interesting to fuse vision based localisation, with GPS signal and inertial measurements.

An aspect that has not been addressed in this work is the region of validity of a single sphere. As it was shown in the experimental results, the proposed approach is able to perform online localisation with respect to a reference sphere up to several meters. It would be interesting to quantify this region in order to improve it, for example by taking into account the changes resolution in the warping functions. This could also allow a better compression of the graph of spheres.

Another important feature that will be studied is life long mapping, since the environment is not static it is necessary to update the database with new geometric and photometric information gathered during the online phase. This could be achieved using an online SLAM technique.

\section{Acknowledgements}

This work has been supported by ANR (French National Agency) CityVIP project under grant ANR07_TSFA-013-01. The authors would like to thanks Alexandre Chapoulie for helpful discussions and for his work on loop closure detection.

\section{Appendix}

\subsection{Warping functions}

\section{Perspective projection}

A $3 \mathrm{D}$ point $\mathbf{v} \in \mathbb{R}^{3}$ is projected onto the normalized image plane such that

$$
\overline{\mathbf{p}}=\left[\begin{array}{l}
u \\
v \\
1
\end{array}\right]=\frac{\mathbf{M} \overline{\mathbf{v}}}{\mathbf{e}_{3}^{T} \mathbf{M} \overline{\mathbf{v}}},
$$


where $\overline{\mathbf{v}}=(x, y, z, 1)$ is the homogeneous version of $\mathbf{v}, \mathbf{e}_{3}=\left[\begin{array}{lll}0 & 0 & 1\end{array}\right]^{T}$ is a unit vector allowing to normalize the pixels coordinates with respect to their third component, and $\mathbf{M}$ is the $3 \times 4$ perspective projection matrix defined as

$$
\mathbf{M}=\mathbf{K}\left[\begin{array}{ll}
\mathbf{R} & \mathbf{t}
\end{array}\right]
$$

with $\mathbf{K}$ the $3 \times 3$ intrinsic matrix of the camera and $\mathbf{T}=(\mathbf{R}, \mathbf{t}) \in \mathbb{S E}(3)$ is a rigid transformation.

\section{Spherical projection}

A $3 \mathrm{D}$ point $\mathbf{v} \in \mathbb{R}^{3}$ can be projected onto the unit sphere by

$$
\mathbf{q}_{E}=\left[\begin{array}{c}
x_{S} \\
y_{S} \\
z_{S}
\end{array}\right]=\frac{\mathbf{R v}+\mathbf{t}}{\|\mathbf{R v}+\mathbf{t}\|}
$$

where $\mathbf{T}=(\mathbf{R}, \mathbf{t}) \in \mathbb{S E}(3)$ is a rigid transformation. The point $\mathbf{q}_{E}$ can therefore be converted to spherical coordinates by

$$
\mathbf{q}_{S}=\left[\begin{array}{l}
\theta \\
\phi \\
\rho
\end{array}\right]=\left[\begin{array}{c}
\arctan \left(z_{S} / x_{S}\right) \\
\arctan \left(y_{S} / \sqrt{x_{S}^{2}+z_{S}^{2}}\right) \\
\sqrt{z_{S}^{2}+y_{S}^{2}+z_{S}^{2}}
\end{array}\right] .
$$

\subsection{Jacobians}

The Jacobian matrix of error (11) can be decomposed in 3 Jacobian matrices.

$$
\mathbf{J}(\tilde{\mathbf{x}})=\mathbf{J}_{\mathcal{I}} \mathbf{J}_{w} \mathbf{J}_{\mathbf{T}}
$$

where $\mathbf{J}_{\mathbf{T}}=\frac{\partial \mathbf{T}(\mathbf{x})}{\partial \mathbf{x}}$ is the derivative of equation (1) with respect to $\mathbf{x}$ of dimensions $12 \times 6 . \mathbf{J}_{w}=\frac{\partial \mathbf{w}(\cdot)}{\partial \mathbf{T}}$ is the derivative of the spherical warping with respect to $\mathbf{T}$ of dimensions $3 n m \times 12$ and $\mathbf{J}_{\mathcal{I}}=\frac{\partial \mathcal{I}}{\partial \mathbf{q}_{s}}$ is the spherical image gradient of dimensions $n m \times 3 n m$.

For the multi-sphere localisation of equation (19), the global Jacobian matrix is obtained by stacking the Jacobian of each error, leading to

$$
\mathbf{J}_{G}=\left[\begin{array}{c}
\mathbf{J}_{0} \\
\mathbf{J}_{1} \mathbf{J}_{v}\left(\mathbf{T}_{\mathcal{G}}^{1}\right)
\end{array}\right],
$$


where $\mathbf{J}_{v}\left(\mathbf{T}_{\mathcal{G}}^{1}\right)$ is the adjoint map associated to the pose $\mathbf{T}_{\mathcal{G}}^{1}$, and is defined by

$$
\mathbf{J}_{v}(\mathbf{T})=\left[\begin{array}{cc}
\mathbf{R} & \mathbf{t}_{\times} \mathbf{R} \\
\mathbf{0}_{3} & \mathbf{R}
\end{array}\right]
$$

\subsection{Robust estimation}

The robust diagonal matrix $\mathbf{D}$ is defined such that

$$
\mathbf{D}=\left[\begin{array}{cccc}
w_{1} & 0 & \ldots & 0 \\
0 & w_{2} & \ldots & 0 \\
\vdots & \vdots & \ddots & \vdots \\
0 & 0 & \ldots & w_{n}
\end{array}\right]
$$

where the weights $w_{i} \in[0 ; 1]$ are obtained by:

$$
w_{i}=\frac{\psi\left(\delta_{i} / \sigma\right)}{\delta_{i} / \sigma}, \quad \psi(u)= \begin{cases}u & , \text { if }|u| \leq a \\ a \frac{u}{|u|} & , \text { if }|u|>a\end{cases}
$$

$\delta_{i}$ is the centred residual of each component of the error vector $\mathbf{e}=\left[e_{1}, e_{2}, \ldots, e_{n m}\right]^{\top}$, with $\delta_{i}=e_{i}-$ Median(e), and $\psi$ is the Huber influence function. The proportionality factor for the Huber function is $a=1.345$, which represents $95 \%$ of efficiency under a Gaussian noise.

The values $\delta_{i}$ are normalized by a robust measure of the scale of the distribution (MAD) such that

$$
\sigma=\frac{1}{\Phi^{-1}(0.75)} \operatorname{Median}\left(\left|\delta_{i}-\operatorname{Median}(\delta)\right|\right)
$$

where $\Phi(\cdot)$ is the cumulative distribution function and $1 / \Phi^{-1}(0.75)=1.48$ is the standard deviation for a normal distribution.

\subsection{Multimedia file}

The video attached to this paper illustrates each aspect of the proposed dense mapping and autonomous navigation approach as follows 
- (08s-14s) Spherical acquisition system mounted onto an electric Cycab vehicle.

- $(14 \mathrm{~s}-1 \mathrm{~m})$ Incremental sphere mapping process. Left, current RGB-D sphere. Top right, estimated trajectory and reference sphere positions. Bottom right, new sphere selection criterion.

- $(1 \mathrm{~m}-2 \mathrm{~m})$ Photo-realistic virtual navigation (see Section 6.3.2).

- $(2 \mathrm{~m}-3 \mathrm{~m} 18 \mathrm{~s})$ Online localisation results in the XII ${ }^{t h}$ district of Paris (see Section 6.3.4). Top right, current monocular image. Top middle, closest reference key-frame. Bottom right, virtual view of the model rendered at the current camera pose. Bottom left, current camera pose and key-frames poses.

- (3m18-4m53s) Autonomous navigation results in Clermont Ferrand (see Section 6.4.4).

\section{References}

Anguelov, D., Dulong, C., Filip, D., Frueh, C., Lafon, S., Lyon, R., Ogale, A., Vincent, L., and Weaver, J. (2010). Google street view: Capturing the world at street level. Computer, 43.

Arican, Z. and Frossard, P. (2007). Dense disparity estimation from omnidirectional images. In Proceedings of the IEEE Conference on Advanced Video and Signal Based Surveillance (AVSS), London, UK.

Audras, C., Comport, A. I., Meilland, M., and Rives, P. (2011). Real-time appearance-based slam for rgb-d sensors. In Proceedings of the Australian Conference on Robotics and Automation (ACRA), Melbourne, Australia.

Avidan, S. and Shashua, A. (1997). Novel view synthesis in tensor space. In Proceedings of the IEEE International Conference on Computer Vision and Pattern Recognition (CVPR), San Juan, Puerto Rico.

Baker, P., Fermuller, C., Aloimonos, Y., and Pless, R. (2001). A spherical eye from multiple cameras. In Proceedings of the IEEE International Conference on Computer Vision and Pattern Recognition (CVPR), Kauai, HI, USA.

Baker, S. and Matthews, I. (2001). Equivalence and efficiency of image alignment algorithms. In Proceedings of the IEEE International Conference on Computer Vision and Pattern Recognition (CVPR), Kauai, HI, USA. 
Benhimane, S., Ladikos, A., Lepetit, V., and Navab, N. (2007). Linear and quadratic subsets for templatebased tracking. In Proceedings of the IEEE International Conference on Computer Vision and Pattern Recognition (CVPR), Minneapolis, USA.

Benhimane, S. and Malis, E. (2004). Real-time image-based tracking of planes using efficient second-order minimization. In Proceedings of the IEEE International Conference on Intelligent Robots and Systems (IROS), Sendai, Japan.

Benhimane, S., Malis, E., Rives, P., and Azinheira, J. (2005). Vision-based control for car platooning using homography decomposition. In Proceedings of the IEEE International Conference on Robotics and Automation (ICRA), Barcelona, Spain.

Brown, D. C. (1971). Close-range camera calibration. Photogrammetric engineering, 37(8):855-866.

Burt, P. J. and Adelson, E. H. (1983). A multiresolution spline with application to image mosaics. ACM Transactions on Graphics, 2:217-236.

Cappelle, C., El Najjar, M., Charpillet, F., and Pomorski, D. (2011). Virtual 3d city model for navigation in urban areas. Journal of Intelligent and Robotic Systems, 66(3):1-23.

Caron, G., Marchand, E., and Mouaddib, E. (2011). Tracking planes in omnidirectional stereovision. In Proceedings of the IEEE International Conference on Robotics and Automation (ICRA), Shanghai, China.

Chapoulie, A., Rives, P., and Filliat, D. (2011). A spherical representation for efficient visual loop closing. In Proceedings of the 11th workshop on Omnidirectional Vision, Camera Networks and Non-classical Cameras (OMNIVIS), Barcelona, Spain.

Cobzas, D., Zhang, H., and Jagersand, M. (2003). Image-based localization with depth-enhanced image map. In Proceedings of the IEEE International Conference on Robotics and Automation (ICRA), Taipei, Taiwan.

Comport, A. I., Malis, E., and Rives, P. (2007). Accurate quadrifocal tracking for robust 3d visual odometry. In Proceedings of the IEEE International Conference on Robotics and Automation (ICRA), Rome, Italy.

Comport, A. I., Malis, E., and Rives, P. (2010). Real-time quadrifocal visual odometry. The International Journal of Robotics Research, 29(2-3):245-266. 
Comport, A. I., Marchand, E., Pressigout, M., and Chaumette, F. (2006). Real-time markerless tracking for augmented reality: the virtual visual servoing framework. IEEE Transactions on Visualization and Computer Graphics, 12(4):615-628.

Corke, P., Paul, R., Churchill, W., and Newman, P. (2013). Dealing with shadows: Capturing intrinsic scene appearance for image-based outdoor localisation. In Proceedings of the IEEE International Conference on Intelligent Robots and Systems (IROS), Tokyo, Japan.

Courbon, J., Mezouar, Y., and Martinet, P. (2008). Indoor navigation of a non-holonomic mobile robot using a visual memory. Autonomous Robots, 25:253-266.

Courbon, J., Mezouar, Y., and Martinet, P. (2009). Autonomous navigation of vehicles from a visual memory using a generic camera model. Intelligent Transport System, 10:392-402.

Craciun, D., Paparoditis, N., and Schmitt, F. (2010). Multi-view scans alignment for 3d spherical mosaicing in large-scale unstructured environments. Computer Vision and Image Understanding, 114(11):12481263. Special issue on Embedded Vision.

Cummins, M. and Newman, P. (2008). FAB-MAP: Probabilistic Localization and Mapping in the Space of Appearance. The International Journal of Robotics Research, 27(6):647-665.

Dame, A. and Marchand, E. (2010). Accurate real-time tracking using mutual information. In Proceedings of the IEEE/ACM ACM International Symposium on Mixed and Augmented Reality (ISMAR), Seoul, Korea.

Davison, A. and Murray, D. (2002). Simultaneous localization and map-building using active vision. IEEE Transactions on Pattern Analysis and Machine Intelligence, 24(7):865-880.

Debevec, P. E., Taylor, C. J., and Malik, J. (1996). Modeling and rendering architecture from photographs: a hybrid geometry- and image-based approach. In Proceedings of the Annual Conference on Computer Graphics and Interactive Techniques (SIGGRAPH), New Orleans, LA, USA.

Dellaert, F. and Collins, R. (1999). Fast image-based tracking by selective pixel integration. In Proceedings of the International Conference on Computer Vision Workshop on Frame-Rate Vision (ICCV-W), Kerkyra, Corfu, Greece.

Drummond, T., Society, I. C., and Cipolla, R. (2002). Real-time visual tracking of complex structures. IEEE Transactions on Pattern Analysis and Machine Intelligence, 24:932-946. 
Durrant-Whyte, H. and Bailey, T. (2006). Simultaneous localisation and mapping (slam): Part i the essential algorithms. IEEE Robotics and Automation Magazine, 13(2):99-110.

Earthmine (2009). Earthmine spherical sensor. http://www.earthmine.com.

Furukawa, Y. and Ponce, J. (2010). Accurate, dense, and robust multiview stereopsis. IEEE Transactions on Pattern Analysis and Machine Intelligence, 32:1362-1376.

Gallegos, G., Meilland, M., Rives, P., and Comport, A. I. (2010). Appearance-based slam relying on a hybrid laser/omnidirectional sensor. In Proceedings of the IEEE International Conference on Intelligent Robots and Systems (IROS), Taipei, Taiwan.

Geiger, A., Roser, M., and Urtasun, R. (2010). Efficient large-scale stereo matching. In Proceedings of the Asian Conference on Computer Vision (ACCV), Daejeon, Korea.

Goncalves, N. and Araujo, H. (2004). Projection model, 3d reconstruction and rigid motion estimation from non-central catadioptric images. In Proceedings of the International Symposium on 3D Data Processing, Visualization and Transmission (3DPVT), Thessaloniki, Greece.

Gorski, K. M., Hivon, E., Banday, A. J., Wandelt, B. D., Hansen, F. K., Reinecke, M., and Bartelman, M. (2005). Healpix - a framework for high resolution discretization, and fast analysis of data distributed on the sphere. The Astrophysical Journal, 622:759-773.

Gortler, S. J., Grzeszczuk, R., Szeliski, R., and Cohen, M. F. (1996). The lumigraph. In Proceedings of the Annual Conference on Computer Graphics and Interactive Techniques (SIGGRAPH), New Orleans, LA, USA.

Grisetti, G., Grzonka, S., Stachniss, C., Pfaff, P., and Burgard, W. (2007). Efficient estimation of accurate maximum likelihood maps in 3d. In Proceedings of the IEEE International Conference on Intelligent Robots and Systems (IROS), Rome, Italy.

Grisetti, G., Stachniss, C., Grzonka, S., and Burgard, W. (2009). Toro - tree-based network optimizer. http://openslam.org/toro.html.

Guizzo, E. (2011). Google car. http://spectrum.ieee.org/automaton/robotics/artificial-intelligence/howgoogle-self-driving-car-works.

Hager, G. and Belhumeur, P. (1998). Efficient region tracking with parametric models of geometry and illumination. IEEE Transactions on Pattern Analysis and Machine Intelligence, 20(10):1025 -1039. 
Hammoudi, K., Dornaika, F., Soheilian, B., and Paparoditis, N. (2010). Generating raw polygons of street facades from a $2 \mathrm{~d}$ urban map and terrestrial laser range data. In Proceedings of the SSSI Australasian Remote Sensing and Photogrammetry Conference (ARSPC), Alice Springs, NT, Australia.

Handa, A., Newcombe, R. A., Angeli, A., and Davison, A. J. (2012). Real-Time Camera Tracking: When is High Frame-Rate Best? In Proceedings of the European Conference on Computer Vision (ECCV), Florence, Italy.

Harris, C. and Stephens, M. (1988). A combined corner and edge detector. In Proceedings of the 4th Alvey Vision Conference.

He, L., Luo, C., Geng, Y., Zhu, F., and Hao, Y. (2007). Reliable depth map regeneration via a novel omnidirectional stereo sensor. In Proceedings of the 3rd international conference on Advances in visual computing - Volume Part I.

Henry, P., Krainin, M., Herbst, E., Ren, X., and Fox, D. (2010). Rgb-d mapping: Using depth cameras for dense 3d modeling of indoor environments. In Proceedings of the International Symposium on Experimental Robotics (ISER), New Delhi and Agra, India.

Hirschmuller, H. (2008). Stereo processing by semiglobal matching and mutual information. IEEE Transactions on Pattern Analysis and Machine Intelligence, 30:328-341.

Howard, A. (2008). Real-time stereo visual odometry for autonomous ground vehicles. In Proceedings of the IEEE International Conference on Intelligent Robots and Systems (IROS), Nice, France.

Huber, P. (1981). Robust Statistics. New york, Wiley.

Irani, M. and Anandan, P. (1998). Robust multi-sensor image alignment. In International Conference on Computer Vision, Bombay, India.

Irani, M. and Anandan, P. (2000). About direct methods. Vision Algorithms: Theory and Practice, 1883:267277.

Irschara, A., Zach, C., Frahm, J.-M., and Bischof, H. (2009). From structure-from-motion point clouds to fast location recognition. In Proceedings of the IEEE International Conference on Computer Vision and Pattern Recognition (CVPR), Ft. Collins, CO, USA.

Jogan, M. and Leonardis, A. (2000). Robust localization using panoramic view-based recognition. In Proceedings of the IEEE International Conference on Computer Vision and Pattern Recognition (CVPR), Hilton Head, SC, USA. 
Kitt, B., Geiger, A., and Lategahn, H. (2010). Visual odometry based on stereo image sequences with ransac-based outlier rejection scheme. In Proceedings of the IEEE Intelligent Vehicles Symposium (IV), La Jolla, CA, USA.

Klein, G. and Murray, D. (2007). Parallel tracking and mapping for small ar workspaces. In Proceedings of the IEEE/ACM International Symposium on Mixed and Augmented Reality (ISMAR), Nara, Japan.

Klingner, B., Martin, D., and Roseborough, J. (2013). Street view motion-from-structure-from-motion. In Proceedings of the International Conference on Computer Vision (ICCV), Sydney, Australia.

Kolmogorov, V. and Zabih, R. (2001). Computing visual correspondence with occlusions using graph cuts. In Proceedings of the International Conference on Computer Vision (ICCV), Vancouver, Canada.

Konolige, K. and Agrawal, M. (2008). Frameslam: from bundle adjustment to realtime visual mapping. IEEE Transactions on Robotics, 24(5):1066-1077.

Kutulakos, K. N. and Seitz, S. M. (2000). A theory of shape by space carving. International Journal of Computer Vision, 38:307-314.

Lafarge, F. and Mallet, C. (2011). Building large urban environments from unstructured point data. In Proceedings of the International Conference on Computer Vision (ICCV), Barcelona, Spain.

Levoy, M. and Hanrahan, P. (1996). Light field rendering. In Proceedings of the Annual Conference on Computer Graphics and Interactive Techniques (SIGGRAPH), New Orleans, LA, USA.

Lothe, P., Bourgeois, S., Dekeyser, F., Royer, E., and Dhome, M. (2010). Monocular slam reconstructions and 3d city models: Towards a deep consistency. Computer Vision, Imaging and Computer Graphics. Theory and Applications, 68:201-214.

Lovegrove, S. and Davison, A. (2010). Real-time spherical mosaicing using whole image alignment. In Proceedings of the European Conference on Computer Vision (ECCV), Heraklion, Crete, Greece.

Lowe, D. (2004). Distinctive image features from scale-invariant keypoints. International Journal of Computer Vision, 60(2):91-110.

Lowe, D. G. (1991). Fitting parameterized three-dimensional models to images. IEEE Transactions on Pattern Analysis and Machine Intelligence, 13:441-450.

Lucas, B. D. and Kanade, T. (1981). An iterative image registration technique with an application to stereo vision. In Proceedings of the International joint conference on Artificial intelligence (IJCAI), Vancouver, Canada. 
Lui, W. L. D. and Jarvis, R. (2010). Eye-full tower: A gpu-based variable multibaseline omnidirectional stereovision system with automatic baseline selection for outdoor mobile robot navigation. Robotics and Autonomous Systems, 58(6):747-761.

Malis, E. (2004). Improving vision-based control using efficient second-order minimization techniques. In Proceedings of the IEEE International Conference on Robotics and Automation (ICRA), New Orleans, LA, USA.

Marchand, E., Bouthemy, P., and Chaumette, F. (2001). A 2d-3d model-based approach to real-time visual tracking. Image and Vision Computing, 19(13):941-955.

Mei, C., Benhimane, S., Malis, E., and Rives, P. (2006). Constrained multiple planar template tracking for central catadioptric cameras. In Proceedings of the British Machine Vision Conference (BMVC), Edinburgh, UK.

Mei, C., Sibley, G., Cummins, M., Newman, P., and Reid, I. (2010). Rslam: A system for large-scale mapping in constant-time using stereo. International Journal of Computer Vision, 94:198-214. Special issue of BMVC.

Meilland, M., Comport, A. I., and Rives, P. (2010). A spherical robot-centered representation for urban navigation. In Proceedings of the IEEE International Conference on Intelligent Robots and Systems (IROS), Taipei, Taiwan.

Meilland, M., Comport, A. I., and Rives, P. (2011a). Dense visual mapping of large scale environments for real-time localisation. In Proceedings of the IEEE International Conference on Intelligent Robots and Systems (IROS), San Fransisco, USA.

Meilland, M., Comport, A. I., and Rives, P. (2011b). Real-time dense visual tracking under large lighting variations. In Proceedings of the British Machine Vision Conference (BMVC), Dundee, UK.

Meilland, M., Rives, P., and Comport, A. I. (2012). Dense rgb-d mapping for real-time localisation and navigation. In Proceedings of the IEEE Intelligent Vehicles Workshop on Navigation, Positioning and Mapping $(I V-W)$, Alcalá de Henares, Spain.

Micusik, B. and Pajdla, T. (2004). Autocalibration 3d reconstruction with non-central catadioptric cameras. In Proceedings of the IEEE International Conference on Computer Vision and Pattern Recognition (CVPR), Washington, USA. 
Montemerlo, M., Thrun, S., Koller, D., and Wegbreit, B. (2002). FastSLAM: A factored solution to the simultaneous localization and mapping problem. In Proceedings of the National Conference on Artificial Intelligence (AAAI), Edmonton, Canada.

Mouragnon, E., Lhuillier, M., Dhome, M., Dekeyser, F., and Sayd, P. (2006). Real time localization and 3d reconstruction. In Proceedings of the IEEE International Conference on Computer Vision and Pattern Recognition (CVPR), New York, USA.

Nayar, S. (1997). Catadioptric omnidirectional camera. In Proceedings of the IEEE International Conference on Computer Vision and Pattern Recognition (CVPR), San Juan, Puerto Rico.

Newcombe, R. A., Izadi, S., Hilliges, O., Molyneaux, D., Kim, D., Davison, A. J., Kohli, P., Shotton, J., Hodges, S., and Fitzgibbon, A. (2011a). Kinectfusion: Real-time dense surface mapping and tracking. In Proceedings of the IEEE/ACM ACM International Symposium on Mixed and Augmented Reality (ISMAR), Basel, Switzerland.

Newcombe, R. A., Lovegrove, S., and Davison, A. J. (2011b). Dtam: Dense tracking and mapping in realtime. In Proceedings of the International Conference on Computer Vision (ICCV), Barcelona, Spain.

Nistér, D., Naroditsky, O., and Bergen, J. (2004). Visual odometry. In Proceedings of the IEEE International Conference on Computer Vision and Pattern Recognition (CVPR), Washington, USA.

Ogale, A. S. and Aloimonos, Y. (2005). Shape and the stereo correspondence problem. International Journal on Computer Vision, 65(3):147-162.

Olson, E., Leonard, J., and Teller, S. (2006). Fast iterative alignment of pose graphs with poor initial estimates. In Proceedings of the IEEE International Conference on Robotics and Automation (ICRA), Orlando, USA.

Ragot, N., Rossi, R., Savatier, X., Ertaud, J. Y., and Mazari, B. (2008). 3d volumetric reconstruction with a catadioptric stereovision sensor. In Proceedings of the IEEE International Symposium on Industrial Electronics (ISIE), Cambridge, UK.

Royer, E., Lhuillier, M., Dhome, M., and Chateau, T. (2005). Localization in urban environments: Monocular vision compared to a differential gps sensor. In Proceedings of the IEEE International Conference on Computer Vision and Pattern Recognition (CVPR), San Diego, USA.

Shan, Q., Adams, R., Curless, B., Furukawa, Y., and Seitz, S. M. (2013). The Visual Turing Test for Scene Reconstruction. In Proceedings of the International Conference on 3D Vision (3DIMPV), Seattle, USA. 
Shi, J. and Tomasi, C. (1994). Good features to track. In Proceedings of the IEEE International Conference on Computer Vision and Pattern Recognition (CVPR), Seattle, USA.

Sibley, G., Matthies, L., and Sukhatme, G. (2008). A Sliding Window Filter for Incremental SLAM, volume 8 of Lecture Notes in Electrical Engineering. Springer US.

Silveira, G., Malis, E., and Rives, P. (2008). An efficient direct approach to visual SLAM. IEEE Transactions on Robotics, 24(5):969-979.

Spinello, L. and Arras, K. O. (2011). People detection in rgb-d data. In Proceedings of the IEEE International Conference on Intelligent Robots and Systems (IROS), San Fransisco, USA.

Szeliski, R. (2006). Image alignment and stitching: a tutorial. Foundations and Trends in Computer Graphics and Vision, 2(1):1-104.

Tardif, J.-P., George, M., Laverne, M., Kelly, A., and Stentz, A. (2010). A new approach to vision-aided inertial navigation. In Proceedings of the IEEE International Conference on Intelligent Robots and Systems (IROS), Taipei, Taiwan.

Thrun, S. (2002). Probabilistic robotics. ACM Communications, 45:52-57.

Tola, E., Lepetit, V., and Fua, P. (2010). Daisy: an efficient dense descriptor applied to wide baseline stereo. IEEE Transactions on Pattern Analysis and Machine Intelligence, 32(5):815-830.

Triggs, B., Mclauchlan, P. F., Hartley, R. I., and Fitzgibbon, A. W. (2000). Bundle Adjustment - A Modern Synthesis, volume 1883. Lecture Notes in Computer Science.

Vacchetti, L., Lepetit, V., and Fua, P. (2004). Stable Real-Time 3D Tracking using Online and Offline Information. IEEE Transactions on Pattern Analysis and Machine Intelligence, 26(10):1385-1391.

Viola, P. and Wells, W.M., I. (1995). Alignment by maximization of mutual information. In Proceedings of the International Conference on Computer Vision (ICCV), Boston, USA. 